\title{
Discrete maximum principles for nonlinear parabolic PDE systems *
}

\author{
István Faragó ${ }^{1}$, János Karátson ${ }^{1}$, Sergey Korotov ${ }^{2}$ \\ May 12, 2011
}

\author{
${ }^{1}$ Department of Applied Analysis and Computational Mathematics, Eötvös Loránd \\ University; H-1518, Budapest, Pf. 120, Hungary, e-mail: \{farago, karatson\}@cs.elte.hu \\ ${ }^{2}$ BCAM - Basque Center for Applied Mathematics \\ Bizkaia Technology Park, Building 500 \\ E-48160, Derio, Basque Country, Spain, e-mail: korotov@bcamath.org
}

\begin{abstract}
Discrete maximum principles are established for finite element approximations of nonlinear parabolic PDE systems with mixed boundary and interface conditions. The results are based on an algebraic discrete maximum principle for suitable ODE systems.

Keywords: Nonlinear parabolic system, discrete maximum principle, finite element method

Mathematics Subject Classification: 65N30,65N50,65M60, 65M22
\end{abstract}

\section{Introduction}

The numerical solution of parabolic partial differential equations or systems is a widespread task in numerical analysis, see, e.g., [29, 30, 32]. The discrete solution is naturally required to reproduce the basic qualitative properties of the exact solution. Such a property for parabolic equations is the (continuous) maximum principle (CMP), see e.g. [14, 28] for its several variants. Its discrete analogues, the so-called discrete maximum principles (DMPs) for linear parabolic problems were first presented in the papers [15, 25], and later developed and analysed in many papers, see e.g. $[9,10,31]$ and the references therein. A related important discrete qualitative property is the so-called nonnegativity preservation, analysed in the context of DMPs e.g. in [9].

It is well-known from the above works on linear parabolic equations that the usual relation between the space and time discretization steps is generally

$$
\Delta t=O\left(h^{2}\right)
$$

* Supported by the Hungarian Research Grant OTKA No.K 67819, by HAS under the Bolyai János Scholarship, Grant MTM2008-03541 of the MICINN, Spain, the ERC Advanced Grant FP7-246775 NUMERIWAVES, and Grant PI2010-04 of the Basque Government. 
(i.e., the ratio of $\Delta t$ and $O\left(h^{2}\right)$ should remain between two positive constants as they tend to 0$)$, both to achieve convergence and to satisfy the DMP $[9,10]$. We note that mass lumping can be used to avoid the lower bound $\Delta t \geq c h^{2}$ (which requires sufficiently large time-steps w.r.t. $h^{2}$ ), see $[15,33,34]$; on the other hand, the really important restriction is not the large time steps but the sufficiently small time steps w.r.t. $h^{2}$ (i.e. the upper bound $\Delta t \leq c h^{2}$ ), which is however inevitable in any work even for linear DMP $[9,10]$. The other main assumption to achieve the DMP arises for the space mesh. When using FEM, one has to impose certain geometrical restrictions, e.g. for simplicial elements this means certain acuteness of the mesh in the presence of lower order terms. These conditions also appear in the widely studied elliptic case, see, e.g., [5, 15, 16, 22, 26, 27, 38, 41] and the references therein. A fairly general algebraic condition on the FE basis functions that covers most of these conditions has been given in [24]:

$$
\nabla \varphi_{i} \cdot \nabla \varphi_{j} \leq 0 \text { on } \Omega \text { and } \int_{\Omega} \nabla \varphi_{i} \cdot \nabla \varphi_{j} \leq-K_{0} h^{d-2}
$$

for all $i, j$, where $h$ is the mesh size, $d$ is the space dimension and $K_{0}>0$ is a constant (independent of $h$ ). Under such conditions, the DMP holds for small enough $h$, namely, for $h<h_{0}$ where $h_{0}$ is a computable bound.

In this paper we prove that proper discrete maximum principles hold for nonlinear parabolic systems of PDEs, discretized in space by FEM, under the same conditions as discussed above. To our knowledge, there have appeared very few papers on nonlinear equations concerning parabolic DMP. A related result in [8, Th. 5.13] shows that FEM for some semilinear reaction-diffusion systems on $2 \mathrm{D}$ domains preserves invariant regions under certain assumptions, which is closely related to DMP. Some results on DMP for FEM for certain nonlinear parabolic equations have been given in [13]. Our goal is to extend the result of [13] to systems as general as possible, involving nonsymmetric terms and mixed boundary and interface conditions as well. The coupling of the equations in the system is cooperative and weakly diagonally dominant, similarly to the elliptic case [24].

The CMP itself has been extended for nonlinear parabolic systems of PDEs in different forms, often in the context of invariant sets, see, e.g., [7, 39, 40]. We find it natural to require an analogy of the DMP, known for linear equations, to hold for nonlinear systems as well. First, this is suggested by the physical meaning of such systems, most often in the special form of nonnegativity of the solution. Second, in the elliptic case the same CMP holds for related nonlinear equations as for linear equations [22], and a natural analogue of these holds for systems [24].

An important step in our process is to establish a purely algebraic DMP for systems of ordinary differential equations (ODEs), to which our results on PDE systems can then be reduced. This DMP for ODEs is of independent interest, and can be regarded as a basic property that underlies parabolic PDEs. This is analogous to the algebraic or matrix maximum principle for generalized nonnegative matrices $[4,37]$ that underlies most elliptic DMP results.

The paper is organized as follows. In Section 2, we formulate the considered class of systems. The discretization scheme is given in detail in Section 3. Section 4 is devoted to 
62

the algebraic DMP for ODE systems. The DMP and related nonnegativity preservation for the considered parabolic systems are presented in Section 5. Finally, various examples are given in Section 6.

\section{The class of problems}

In this paper we consider the following type of nonlinear parabolic systems, involving cooperative and weakly diagonally dominant coupling, nonsymmetric terms and mixed boundary and interface conditions. Find a function $u=u(x, t)=\left(u_{1}(x, t), \ldots, u_{s}(x, t)\right)$ such that for all $k=1, \ldots, s$,

$$
\frac{\partial u_{k}}{\partial t}-\operatorname{div}\left(a_{k}(x, t, u, \nabla u) \nabla u_{k}\right)+\mathbf{w}_{k}(x, t) \cdot \nabla u_{k}+q_{k}(x, t, u)=f_{k}(x, t)
$$

$$
\text { in } Q_{T}:=\left(\Omega \backslash \Gamma_{\text {int }}\right) \times(0, T) \text {, }
$$

where $\Omega$ is a bounded domain in $\mathbf{R}^{d}$ and $T>0$, further, the boundary, interface and initial conditions are as follows $(k=1, \ldots, s)$ :

$$
u_{k}(x, t)=g_{k}(x, t) \quad \text { for } \quad(x, t) \in \Gamma_{D} \times[0, T],
$$

$$
\begin{gathered}
a_{k}(x, t, u, \nabla u) \frac{\partial u_{k}}{\partial \nu}+s_{k}(x, t, u)=\gamma_{k}(x, t) \quad \text { for } \quad(x, t) \in \Gamma_{N} \times[0, T], \\
{\left[u_{k}\right]_{\Gamma_{\text {int }}}=0 \text { and }\left[a_{k}(x, t, u, \nabla u) \frac{\partial u_{k}}{\partial \nu}+s_{k}(x, t, u)\right]_{\Gamma_{i n t}}=\gamma_{k}(x, t)} \\
\text { for } \quad(x, t) \in \Gamma_{\text {int }} \times[0, T],
\end{gathered}
$$

$$
u_{k}(x, 0)=u_{k}^{(0)}(x) \text { for } \quad x \in \Omega,
$$

respectively, where $\nu$ is the outer normal vector and $[.]_{\Gamma_{\text {int }}}$ denotes the jump (i.e., the difference of the limits from the two sides of the interface $\left.\Gamma_{i n t}\right)$ of a function. We impose the following

\section{Assumptions 2.1.}

(A1) (Domain.) $\Omega$ is a bounded polytopic domain in $\mathbf{R}^{d} ; \Gamma_{N}, \Gamma_{D} \subset \partial \Omega$ are disjoint open subsets of $\partial \Omega$ such that $\partial \Omega=\bar{\Gamma}_{D} \cup \bar{\Gamma}_{N}$, and $\Gamma_{\text {int }}$ is a piecewise $C^{1}$ surface in $\Omega$.

(A2) (Smoothness.) For all $k=1, \ldots, s$, the scalar functions $a_{k}: Q_{T} \times \mathbf{R}^{s} \times \mathbf{R}^{d \times s} \rightarrow \mathbf{R}$, $q_{k}: Q_{T} \times \mathbf{R}^{s} \rightarrow \mathbf{R}$ and $s_{k}:\left(\Gamma_{N} \cup \Gamma_{i n t}\right) \times[0, T] \times \mathbf{R}^{s} \rightarrow \mathbf{R}$ are measurable and bounded, further, $q_{k}$ and $s_{k}$ are continuously differentiable w.r.t. their variables in $\mathbf{R}^{s}$, on their domains of definition. Further, $\mathbf{w}_{k} \in W^{1, \infty}\left(Q_{T}\right), f_{k} \in L^{\infty}\left(Q_{T}\right)$, $\gamma_{k} \in L^{2}\left(\left(\Gamma_{N} \cup \Gamma_{\text {int }}\right) \times[0, T]\right), g_{k} \in L^{\infty}\left(\Gamma_{D} \times[0, T]\right)$ and $u_{k}^{(0)} \in L^{\infty}(\Omega)$.

(A3) (Ellipticity for the principal space term.) There exist constants $\mu_{0}$ and $\mu_{1}$ such that

$$
0<\mu_{0} \leq a_{k}(x, t, \xi, \eta) \leq \mu_{1}
$$

for all $k=1, \ldots, s$ and all $(x, t, \xi, \eta) \in \Omega \times(0, T) \times \mathbf{R}^{s} \times \mathbf{R}^{d \times s}$. 
(A4) (Coercivity.) For all $k=1, \ldots, s$, we have $\operatorname{div} \mathbf{w}_{k} \leq 0$ on $\Omega, \mathbf{w}_{k} \cdot \nu \geq 0$ on $\Gamma_{N}$, further, $\left[\mathbf{w}_{k}\right]_{\Gamma_{\text {int }}}=0$ and $\left[\mathbf{w}_{k} \cdot \nu\right]_{\Gamma_{\text {int }}} \geq 0$.

(A5) (Growth.) Let $2 \leq p_{1}$ if $d=2$ and $2 \leq p_{1}<\frac{2 d}{d-2}$ if $d>2$, further, let $2 \leq p_{2}$ if $d=2$ and $2 \leq p_{2}<\frac{2 d-2}{d-2}$ if $d>2$. There exist constants $\alpha_{1}, \alpha_{2}, \beta_{1}, \beta_{2} \geq 0$ such that for any $x \in \Omega$ (or $x \in \Gamma_{N} \cup \Gamma_{\text {int }}$, resp.), $t \in(0, T), \xi \in \mathbf{R}^{s}$, and any $k, l=1, \ldots, s$,

$$
\left|\frac{\partial q_{k}}{\partial \xi_{l}}(x, t, \xi)\right| \leq \alpha_{1}+\beta_{1}|\xi|^{p_{1}-2}, \quad\left|\frac{\partial s_{k}}{\partial \xi_{l}}(x, t, \xi)\right| \leq \alpha_{2}+\beta_{2}|\xi|^{p_{2}-2} .
$$

(A6) (Cooperativity.) For all $k, l=1, \ldots, s, x \in \Omega$ (or $x \in \Gamma_{N} \cup \Gamma_{\text {int }}$, resp.), $t \in(0, T)$, $\xi \in \mathbf{R}^{s}$,

$$
\frac{\partial q_{k}}{\partial \xi_{l}}(x, t, \xi) \leq 0, \quad \frac{\partial s_{k}}{\partial \xi_{l}}(x, t, \xi) \leq 0, \quad \text { whenever } k \neq l
$$

(A7) (Weak diagonal dominance.) For all $k=1, \ldots, s, x \in \Omega$ (or $x \in \Gamma_{N} \cup \Gamma_{\text {int }}$, resp.), $t \in(0, T), \quad \xi \in \mathbf{R}^{s}$,

$$
\sum_{l=1}^{s} \frac{\partial q_{k}}{\partial \xi_{l}}(x, t, \xi) \geq 0 . \quad \sum_{l=1}^{s} \frac{\partial s_{k}}{\partial \xi_{l}}(x, t, \xi) \geq 0
$$

Remark 2.1 Assumptions (A6)-(A7) imply for all $k=1, \ldots, s, x \in \Omega$ (or $x \in \Gamma_{N} \cup \Gamma_{\text {int }}$, resp.), $t \in(0, T), \quad \xi \in \mathbf{R}^{s}$ that $\quad \frac{\partial q_{k}}{\partial \xi_{k}}(x, t, \xi) \geq 0, \quad \frac{\partial s_{k}}{\partial \xi_{k}}(x, t, \xi) \geq 0$.

We will define weak solutions in a usual way. The interface conditions are handled similarly to the Neumann boundary, see e.g. [23]; now we can join these two sets and denote

$$
\Gamma:=\Gamma_{N} \cup \Gamma_{i n t}
$$

in the sequel. Let

$$
H_{D}^{1}(\Omega):=\left\{u \in H^{1}(\Omega): u_{\mid \Gamma_{D}}=0\right\} .
$$

A function $u: Q_{T} \rightarrow \mathbf{R}^{s}$ is called the weak solution of the problem (1)-(5) if for all $k=1, \ldots, s, u_{k}$ are continuously differentiable with respect to $t$ and $u_{k}(., t) \in H_{D}^{1}(\Omega)$ for all $t \in(0, T)$, and satisfy the relation

$\int_{\Omega} \sum_{k=1}^{s} \frac{\partial u_{k}}{\partial t} v_{k} d x+\int_{\Omega} \sum_{k=1}^{s}\left(a_{k}(x, t, u, \nabla u) \nabla u_{k} \cdot \nabla v_{k}+\left(\mathbf{w}_{k}(x, t) \cdot \nabla u_{k}\right) v_{k}+q_{k}(x, t, u) v_{k}\right) d x$ $+\int_{\Gamma} \sum_{k=1}^{s} s_{k}(x, t, u) v_{k} d \sigma=\int_{\Omega} \sum_{k=1}^{s} f_{k} v_{k} d x+\int_{\Gamma} \sum_{k=1}^{s} \gamma_{k} v_{k} d \sigma \quad\left(\forall v \in H_{D}^{1}(\Omega)^{s}, \quad t \in(0, T)\right)$,

further,

$$
u_{k}=g_{k} \quad \text { on } \quad[0, T] \times \Gamma_{D},\left.\quad u_{k}\right|_{t=0}=u_{k}^{(0)} \quad \text { in } \quad \Omega .
$$

${ }_{3}$ Here and in the sequel, equality of functions in Lebesgue or Sobolev spaces is understood almost everywhere. 


\section{Discretization scheme}

The full discretization of problem (1)-(5) is built up from two standard steps in space and time; in addition, suitable vector basis functions are involved.

\subsection{Semidiscretization in space}

Let $\mathcal{T}_{h}$ be a finite element mesh over the solution domain $\Omega \subset \mathbf{R}^{d}$, where $h$ stands for the discretization parameter. We choose basis functions in the following way. First, let $\bar{n}_{0} \leq \bar{n}$ be positive integers and let us choose basis functions

$$
\varphi_{1}, \ldots, \varphi_{\bar{n}_{0}} \in H_{D}^{1}(\Omega), \quad \varphi_{\bar{n}_{0}+1}, \ldots, \varphi_{\bar{n}} \in H^{1}(\Omega) \backslash H_{D}^{1}(\Omega),
$$

which are associated with the homogeneous and inhomogeneous boundary conditions on $\Gamma_{D}$, respectively. These basis functions are assumed to be continuous on $\bar{\Omega}$ and to satisfy

$$
\varphi_{p} \geq 0 \quad(p=1, \ldots, \bar{n}), \quad \sum_{p=1}^{\bar{n}} \varphi_{p} \equiv 1,
$$

further, that there exist node points $B_{p} \in \Omega \cup \Gamma_{N}\left(p=1, \ldots, \bar{n}_{0}\right)$ and $B_{p} \in \Gamma_{D}(p=$ $\left.\bar{n}_{0}+1, \ldots, \bar{n}\right)$ such that

$$
\varphi_{p}\left(B_{q}\right)=\delta_{p q}
$$

where $\delta_{p q}$ is the Kronecker symbol. These conditions hold e.g. for standard linear, bilinear or prismatic finite elements. We note that in general $\bar{n}=O\left(h^{d}\right)$. Further, one usually considers a family of subspaces and lets $h \rightarrow 0$, hence we will stress the independence of $h$ for certain bounds where applicable.

We in fact need a basis in the corresponding product spaces, which we define by repeating the above functions in each of the $s$ coordinates and setting zero in the other coordinates. That is, let $N_{0}:=s \bar{n}_{0}$ and $N:=s \bar{n}$. First, for any $1 \leq i \leq N_{0}$,

$$
\text { if } i=\left(k_{0}-1\right) \bar{n}_{0}+p \text { for some } 1 \leq k_{0} \leq s \text { and } 1 \leq p \leq \bar{n}_{0} \text {, then }
$$

$$
\phi_{i}:=\left(0, \ldots, 0, \varphi_{p}, 0, \ldots, 0\right) \quad \text { where } \varphi_{p} \text { stands at the } k_{0} \text { th entry, }
$$

that is, the $m$ th coordinate of $\phi_{i}$ satisfies $\left(\phi_{i}\right)_{m}=\varphi_{p}$ if $m=k_{0}$ and $\quad\left(\phi_{i}\right)_{m}=0$ if $m \neq k_{0}$. From these, we let

$$
V_{h}^{0}:=\operatorname{span}\left\{\phi_{1}, \ldots, \phi_{N_{0}}\right\} \subset H_{D}^{1}(\Omega)^{s} .
$$

Similarly, for any $N_{0}+1 \leq i \leq N$,

if $i=N_{0}+\left(k_{0}-1\right)\left(\bar{n}-\bar{n}_{0}\right)+p-\bar{n}_{0} \quad$ for some $1 \leq k_{0} \leq s$ and $\bar{n}_{0}+1 \leq p \leq \bar{n}$, then

$$
\phi_{i}:=\left(0, \ldots, 0, \varphi_{p}, 0, \ldots, 0\right)^{T} \quad \text { where } \varphi_{p} \text { stands at the } k_{0} \text { th entry, }
$$

that is, the $m$ th coordinate of $\phi_{i}$ satisfies $\left(\phi_{i}\right)_{m}=\varphi_{p}$ if $m=k_{0}$ and $\left(\phi_{i}\right)_{m}=0$ if $m \neq k_{0}$. From (16) and these, we let

$$
V_{h}:=\operatorname{span}\left\{\phi_{1}, \ldots, \phi_{N}\right\} \subset H^{1}(\Omega)^{s} .
$$


Using the above FEM subspaces, one can define the semidiscrete problem for (10) with initial-boundary conditions (11). We look for a vector function $u_{h}=u_{h}(x, t)$ that satisfies (10) for all $v_{h}=\left(v_{1}, \ldots, v_{s}\right) \in V_{h}^{0}$, and the conditions

$u_{k}^{h}(x, 0)=u_{k}^{(0), h}(x) \quad(x \in \Omega), \quad u_{k}^{h}(., t)-g_{k}^{h}(., t) \in V_{0}^{h} \quad(t \in(0, T))$, for all $k=1, \ldots, s$

must hold. In the above formulae, the functions $u_{k}^{(0), h}$ and $g_{k}^{h}(., t)$ (for any fixed $t$ ) are suitable approximations of the given functions $u_{0}$ and $g(., t)$, respectively. In particular, we will use the following form to describe the $k$ th coordinate $g_{k}^{h}$ :

$$
g_{k}^{h}(x, t)=\sum_{p=1}^{\bar{n}_{\partial}} g_{p}^{(k)}(t) \varphi_{\bar{n}_{0}+p}(x), \quad \text { where } \quad \bar{n}_{\partial}:=\bar{n}-\bar{n}_{0} .
$$

We seek the $k$ th coordinate function $u_{k}$ of the numerical solution in the form

$$
u_{k}^{h}(x, t)=\sum_{p=1}^{\bar{n}} u_{p}^{(k)}(t) \varphi_{p}(x)+g_{k}(x, t)=\sum_{p=1}^{\bar{n}_{0}} u_{p}^{(k)}(t) \varphi_{p}(x)+\sum_{p=1}^{\bar{n}_{\partial}} g_{p}^{(k)}(t) \varphi_{\bar{n}_{0}+p}(x),
$$

where the coefficients $u_{p}^{(k)}(t)\left(p=1, \ldots, \bar{n}_{0}\right)$ are unknown. The set of all coefficient functions will be ordered in the following vector:

$$
\begin{aligned}
\mathbf{u}^{h}(t)=\left(u_{1}^{(1)}(t), \ldots, u_{\bar{n}_{0}}^{(1)}(t) ; u_{1}^{(2)}(t), \ldots, u_{\bar{n}_{0}}^{(2)}(t) ; \ldots ; u_{1}^{(s)}(t), \ldots, u_{\bar{n}_{0}}^{(s)}(t) ;\right. \\
\\
\left.g_{1}^{(1)}(t), \ldots, g_{\bar{n}_{\partial}}^{(1)}(t) ; g_{1}^{(2)}(t), \ldots, g_{\bar{n}_{\partial}}^{(2)}(t) ; \ldots ; g_{1}^{(s)}(t), \ldots, g_{\bar{n}_{\partial}}^{(s)}(t)\right)^{T}
\end{aligned}
$$

(where ${ }^{T}$ denotes the transposed of a vector), that is, $\mathbf{u}^{h}(t)$ has $N_{0}=s \bar{n}_{0}$ coordinates from $u_{1}^{(1)}(t)$ to $u_{\bar{n}_{0}}^{(s)}(t)$ belonging to the points in the interior or on $\Gamma$, and then $N-N_{0}=s\left(\bar{n}-\bar{n}_{0}\right)$ coordinates from $g_{1}^{(1)}(t)$ to $g_{\bar{n}_{\partial}}^{(s)}(t)$ belonging to the boundary points on $\Gamma_{D}$, such that the upper index from 1 to $s$ gives the number of coordinate in the parabolic system. We will also use the notations

$$
\mathbf{u}^{\left(k_{0}\right)}(t):=\left(u_{1}^{\left(k_{0}\right)}(t), \ldots, u_{\bar{n}_{0}}^{\left(k_{0}\right)}(t)\right), \quad \mathbf{g}^{\left(k_{0}\right)}(t):=\left(g_{1}^{\left(k_{0}\right)}(t), \ldots, g_{\bar{n}_{\partial}}^{\left(k_{0}\right)}(t)\right)
$$

for any fixed $k_{0}=1, \ldots, s$, to denote the corresponding sub- $\bar{n}_{0}$-tuples of $\mathbf{u}^{h}(t)$ and sub$\bar{n}_{\partial}$-tuples of $\mathbf{g}^{h}(t)$, respectively.

To find the function $\mathbf{u}^{h}(t)$, first note that it is sufficient that $u_{h}$ satisfies (10) for $v=\phi_{i}$ only $\left(i=1,2, \ldots, N_{0}\right)$. Writing the index $i$ in the following form as before:

$$
i=\left(k_{0}-1\right) \bar{n}_{0}+p \quad \text { for some } 1 \leq k_{0} \leq s \text { and } 1 \leq p \leq \bar{n}_{0},
$$

the function $v=\phi_{i}$ has $k$ th coordinates $v_{k}=\delta_{k, k_{0}} \varphi_{p}$ (where $\delta_{k, k_{0}}$ is the Kronecker symbol) for $k=1, \ldots, s$, hence (10) yields

$$
\int_{\Omega} \frac{\partial u_{k_{0}}}{\partial t} \varphi_{p} d x+\int_{\Omega}\left(a_{k_{0}}(x, t, u, \nabla u) \nabla u_{k_{0}} \cdot \nabla \varphi_{p}+\left(\mathbf{w}_{k_{0}}(x, t) \cdot \nabla u_{k_{0}}\right) \varphi_{p}+q_{k_{0}}(x, t, u) \varphi_{p}\right) d x
$$




$$
+\int_{\Gamma} s_{k_{0}}(x, t, u) \varphi_{p} d \sigma=\int_{\Omega} f_{k_{0}} \varphi_{p} d x+\int_{\Gamma} \gamma_{k_{0}} \varphi_{p} d \sigma \quad\left(1 \leq k_{0} \leq s, 1 \leq p \leq \bar{n}_{0}\right) .
$$

For fixed $k_{0}$, using (20), the first integral in (23) becomes $\overline{\mathbf{M}}\left[\frac{\mathrm{d} \mathbf{u}^{\left(k_{0}\right)}}{\mathrm{d} t}, \frac{\mathrm{d} \mathbf{g}^{\left(k_{0}\right)}}{\mathrm{d} t}\right]$, where

$$
\overline{\mathbf{M}}=\left[M_{p q}\right]_{\bar{n}_{0} \times \bar{n}}, \quad M_{p q}=\int_{\Omega} \varphi_{p} \varphi_{q} d x .
$$

We shall use the corresponding partition

$$
\overline{\mathbf{M}}=\left[\overline{\mathbf{M}}_{0} \mid \overline{\mathbf{M}}_{\partial}\right], \quad \text { where } \quad \overline{\mathbf{M}}_{0} \in \mathbf{R}^{\bar{n}_{0} \times \bar{n}_{0}}, \quad \overline{\mathbf{M}}_{\partial} \in \mathbf{R}^{\bar{n}_{0} \times \bar{n}_{\partial}}
$$

and here $\overline{\mathbf{M}}_{0}$ is the mass matrix corresponding to the interior of $\Omega$. Let $k_{0}=1, \ldots, s$ and let us define the partitioned block matrix

$$
\mathbf{M}:=\left[\operatorname{blockdiag}_{s}\left(\overline{\mathbf{M}}_{0}, \overline{\mathbf{M}}_{0}, \ldots, \overline{\mathbf{M}}_{0}\right) \mid \operatorname{blockdiag}_{s}\left(\overline{\mathbf{M}}_{\partial}, \overline{\mathbf{M}}_{\partial}, \ldots, \overline{\mathbf{M}}_{\partial}\right)\right] \in \mathbf{R}^{N_{0} \times N}
$$

Then we are led to the following Cauchy problem for the system of ordinary differential equations:

$$
\mathbf{M} \frac{\mathrm{d} \mathbf{u}^{h}}{\mathrm{~d} t}+\mathbf{G}\left(t, \mathbf{u}^{h}(t)\right)=\mathbf{f}(t)
$$

$$
\mathbf{u}^{h}(0)=\mathbf{u}_{0}^{h}
$$

where using the form of $i$ as in (22),

$$
\begin{gathered}
\mathbf{G}\left(t, \mathbf{u}^{h}(t)\right)=\left[G\left(t, \mathbf{u}^{h}(t)\right)_{i}\right]_{i=1, \ldots, N_{0}} \\
G\left(t, \mathbf{u}^{h}(t)\right)_{i}=\int_{\Omega}\left(a_{k_{0}}(x, t, u, \nabla u) \nabla u_{k_{0}} \cdot \nabla \varphi_{p}+\left(\mathbf{w}_{k_{0}}(x, t) \cdot \nabla u_{k_{0}}\right) \varphi_{p}+q_{k_{0}}(x, t, u) \varphi_{p}\right) d x \\
+\int_{\Gamma} s_{k_{0}}(x, t, u) \varphi_{p} d \sigma \\
\mathbf{f}(t)=\left[f_{i}(t)\right]_{i=1, \ldots, N_{0}}, \quad f_{i}(t)=\int_{\Omega} f_{k_{0}}(x, t) \varphi_{p}(x) d x+\int_{\Gamma} \gamma_{k_{0}}(x, t) \varphi_{p}(x) d \sigma(x),
\end{gathered}
$$

and finally, $\mathbf{u}_{0}^{h}$ is defined by setting $t=0$ in (21) and using that $u_{p}^{(k)}(0)=u_{k}^{(0)}\left(B_{p}\right)$ for $k=1, \ldots, s$ and $p=1, \ldots, \bar{n}_{0}$.

The solution $\mathbf{u}^{h}=\mathbf{u}^{h}(t)$ of problem (26)-(27) is called the semidiscrete solution. Here the coefficients $g_{p}^{(k)}(t)$ are given, hence (26) can be reduced to a system where $\mathbf{M}$ is replaced by the nonsingular square matrix $\mathbf{M}_{0}:=\operatorname{blockdiag}_{s}\left(\overline{\mathbf{M}}_{0}, \overline{\mathbf{M}}_{0}, \ldots, \overline{\mathbf{M}}_{0}\right)$ only. Then existence and uniqueness for (26)-(27) is ensured by Assumptions 2.1, since then $\mathrm{G}$ is locally Lipschitz continuous. 


\subsection{Full discretization}

In order to get a fully discrete numerical scheme, we choose a time-step $\Delta t$ and denote the approximation to $\mathbf{u}^{h}\left(t_{n}\right)$ and $\mathbf{f}\left(t_{n}\right)$ by $\mathbf{u}^{n}$ and $\mathbf{f}^{n}$ (where $t_{n}:=n \Delta t, \quad n=0,1,2, \ldots, n_{T}$, $T=n_{T} \Delta t$ ), respectively. To discretize (26) in time, we apply the simplest and most commonly used one-step time discretization method, the so-called $\theta$-method $[15,32]$ with some given parameter

$$
\theta \in(0,1]
$$

We note that the case $\theta=0$, which is otherwise also acceptable, will be excluded later by condition (75).

We then obtain a system of nonlinear algebraic equations of the form

$$
\mathbf{M} \frac{\mathbf{u}^{n+1}-\mathbf{u}^{n}}{\Delta t}+\theta \mathbf{G}\left(t_{n+1}, \mathbf{u}^{n+1}\right)+(1-\theta) \mathbf{G}\left(t_{n}, \mathbf{u}^{n}\right)=\mathbf{f}^{(n, \theta)}:=\theta \mathbf{f}^{n+1}+(1-\theta) \mathbf{f}^{n},
$$

$n=0,1, \ldots, n_{T}-1$, which can be rewritten as a recursion

$$
\mathbf{M} \mathbf{u}^{n+1}+\theta \Delta t \mathbf{G}\left(t_{n+1}, \mathbf{u}^{n+1}\right)=\mathbf{M} \mathbf{u}^{n}-(1-\theta) \Delta t \mathbf{G}\left(t_{n}, \mathbf{u}^{n}\right)+\Delta t \mathbf{f}^{(n, \theta)}
$$

with $\mathbf{u}^{0}=\mathbf{u}^{h}(0)$. Furthermore, we will use notations

$$
\mathbf{P}\left(\mathbf{u}^{n+1}\right):=\mathbf{M} \mathbf{u}^{n+1}+\theta \Delta t \mathbf{G}\left(t_{n+1}, \mathbf{u}^{n+1}\right), \quad \mathbf{Q}\left(\mathbf{u}^{n}\right):=\mathbf{M} \mathbf{u}^{n}-(1-\theta) \Delta t \mathbf{G}\left(t_{n}, \mathbf{u}^{n}\right),
$$

respectively. Then, the iteration procedure (29) can be also written as

$$
\mathbf{P}\left(\mathbf{u}^{n+1}\right)=\mathbf{Q}\left(\mathbf{u}^{n}\right)+\Delta t \mathbf{f}^{(n, \theta)} .
$$

Finding $\mathbf{u}^{n+1}$ in (31) requires the solution of a nonlinear algebraic system. Similarly as mentioned before, (31) can be reduced to a system with the first $N_{0}$ coefficients, i.e. $\mathbf{M}$ is replaced by the nonsingular square matrix $\mathbf{M}_{0}:=$ blockdiag $\left(\overline{\mathbf{M}}_{0}, \overline{\mathbf{M}}_{0}, \ldots, \overline{\mathbf{M}}_{0}\right)$ only, since the other coefficients of $\mathbf{u}^{n+1}$ are given from the $g_{p}^{(k)}(t)$. Analogously, $\mathbf{P}$ is replaced by $\mathbf{P}_{0}$. The block mass matrix $\mathbf{M}_{0}$ is positive definite, and it follows from Assumptions 2.1 that $\mathbf{u} \mapsto \mathbf{G}(\mathbf{u})$ has positive semidefinite derivatives. hence by the definition in (30), the function $\mathbf{u} \mapsto \mathbf{P}_{0}(\mathbf{u})$ has regular derivatives. This ensures the unique solvability of (31) and, under standard local Lipschitz conditions on the coefficients, also the convergence of the damped Newton iteration, see e.g. [12].

\section{An algebraic discrete maximum principle for $\mathrm{ODE}$ systems}

An important and widely studied special case of our problem is the linear case, in fact, we wish to recast the nonlinear case to that. In this section we establish an algebraic DMP for systems of ordinary differential equations (ODEs), which can be later used for our discretized parabolic PDE system. 
The motivation for that is the well-known continuous maximum principle (CMP) for a linear parabolic PDE. Consider the problem

$$
\frac{\partial u}{\partial t}-k \Delta u+c(x) u=f(x, t) \quad \text { in } Q_{T}, \quad u=g \quad \text { on }[0, T] \times \partial \Omega,\left.\quad u\right|_{t=0}=u_{0} \text { in } \Omega
$$

where $k>0$ is constant and $c \geq 0$. If the data and solution are assumed to be sufficiently smooth, then problem (32) satisfies the following CMP [11]:

$$
\min \left\{0 ; \min _{\bar{\Gamma}_{t_{1}}} g\right\}+t_{1} \min \left\{0 ; \min _{\bar{Q}_{t_{1}}} f\right\} \leq u\left(x, t_{1}\right) \leq \max \left\{0 ; \overline{\max }_{\bar{t}_{1}} g\right\}+t_{1} \max \left\{0 ; \overline{\max }_{\bar{t}_{1}} f\right\}
$$

for all $x \in \Omega$ and any fixed $t_{1} \in(0, T)$, where $Q_{t_{1}}:=\Omega \times\left[0, t_{1}\right]$, and $\Gamma_{t_{1}}$ denotes the parabolic boundary, i.e., $\Gamma_{t_{1}}:=\left(\partial \Omega \times\left[0, t_{1}\right]\right) \cup(\Omega \times\{0\})$. A related property, which follows from the above [10], is the continuous nonnegativity preservation principle: relations $f \geq 0, g \geq 0$ and $u_{0} \geq 0$ imply $u(x, t) \geq 0$ for all $(x, t) \in Q_{T}$.

In the discrete case, the ODE system (26) for (32) becomes linear and has the form

$$
\mathbf{M} \frac{\mathrm{d} \mathbf{u}^{h}}{\mathrm{~d} t}+\mathbf{K} \mathbf{u}^{h}(t)=\mathbf{f} .
$$

Suitable analogues of (33) have been established e.g. in [11] for such discretized PDEs. Below our goal is to formulate a DMP purely algebraically for such ODE systems, to which our results on PDE systems can then be reduced.

\subsection{The Cauchy problem and its discretization}

Let us consider the Cauchy problem for the system of linear ordinary differential equations

$$
\mathbf{M} \frac{\mathrm{d} \overline{\mathbf{u}}}{\mathrm{d} t}+\mathbf{K} \overline{\mathbf{u}}=\mathbf{f}
$$

where $\mathbf{M}=\left[\mathbf{M}_{0} \mid \mathbf{M}_{\partial}\right], \quad \mathbf{K}=\left[\mathbf{K}_{0} \mid \mathbf{K}_{\partial}\right] \in \mathbf{R}^{N_{0} \times N}$ are partitioned matrices with the entries $\mathbf{M}_{0}, \mathbf{K}_{0} \in \mathbf{R}^{N_{0} \times N_{0}}, \mathbf{M}_{\partial}, \mathbf{K}_{\partial} \in \mathbf{R}^{N_{0} \times N_{\partial}}\left(N=N_{0}+N_{\partial}\right), \mathbf{f}(t) \in \mathbf{R}^{N_{0}}$ for all $t>0$ and $\overline{\mathbf{u}}(0) \in \mathbf{R}^{N}$ are given. Here $\overline{\mathbf{u}}(t) \in \mathbf{R}^{N}$ has the partitioning $[\mathbf{u}(t) \mid \mathbf{g}(t)]^{T}$, where $\mathbf{u}(t) \in \mathbf{R}^{N_{0}}$, $\mathbf{g}(t) \in \mathbf{R}^{N_{\partial}}$ and $\mathbf{g}(t)$ for $t \geq 0$ and $\mathbf{u}(0)$ are given. We seek the unknown function $\mathbf{u}(t)$ for $t>0$.

We impose the following conditions for the matrices $\mathbf{M}$ and $\mathbf{K}$, wherein $i=1, \ldots, N_{0}$, $j=1, \ldots, N$ :

(B1) $\quad K_{i j} \leq 0$ for all $i \neq j$;

$$
\sum_{j=1}^{N} K_{i j} \geq 0 \quad \text { for all } i
$$


Constructing a full discretization of (35) as in subsection 3.2, we obtain a recursion of algebraic systems analogously to (29):

$$
(\mathbf{M}+\theta \Delta t \mathbf{K}) \overline{\mathbf{u}}^{n+1}=(\mathbf{M}-(1-\theta) \Delta t \mathbf{K}) \overline{\mathbf{u}}^{n}+\Delta t \mathbf{f}^{(n, \theta)},
$$

further, the matrices $\mathbf{M}+\theta \Delta t \mathbf{K}$ and $\mathbf{M}-(1-\theta) \Delta t \mathbf{K}$ are denoted by $\mathbf{A}$ and $\mathbf{B}$ respectively. In what follows, we shall use the following partitions of the matrices and vectors:

$$
\mathbf{A}=\left[\mathbf{A}_{0} \mid \mathbf{A}_{\partial}\right], \quad \mathbf{B}=\left[\mathbf{B}_{0} \mid \mathbf{B}_{\partial}\right], \quad \overline{\mathbf{u}}^{n}=\left[\begin{array}{l}
\mathbf{u}^{n} \\
\mathbf{g}^{n}
\end{array}\right],
$$

where, obviously, $\mathbf{A}_{0}$ and $\mathbf{B}_{0}$ are quadratic matrices from $\mathbf{R}^{N_{0} \times N_{0}} ; \mathbf{A}_{\partial}, \mathbf{B}_{\partial} \in \mathbf{R}^{N_{0} \times N_{\partial}}$, $\mathbf{u}^{n}=\left[u_{1}^{n}, \ldots, u_{N_{0}}^{n}\right]^{T} \in \mathbf{R}^{N_{0}}$ and $\mathbf{g}^{n}=\left[g_{1}^{n}, \ldots, g_{N_{\partial}}^{n}\right]^{T} \in \mathbf{R}^{N_{\partial}}$. Then, the iteration (36) can be also written as

$$
\mathbf{A} \overline{\mathbf{u}}^{n+1}=\mathbf{B} \overline{\mathbf{u}}^{n}+\Delta t \mathbf{f}^{(n, \theta)},
$$

or

$$
\left[\mathbf{A}_{0} \mid \mathbf{A}_{\partial}\right]\left[\begin{array}{l}
\mathbf{u}^{n+1} \\
\mathbf{g}^{n+1}
\end{array}\right]=\left[\mathbf{B}_{0} \mid \mathbf{B}_{\partial}\right]\left[\begin{array}{l}
\mathbf{u}^{n} \\
\mathbf{g}^{n}
\end{array}\right]+\Delta t \mathbf{f}^{(n, \theta)}
$$

\subsection{A discrete maximum principle}

Let us use the following notations:

$$
g_{\text {min }}^{n}=\min \left\{g_{1}^{n}, \ldots, g_{N_{\partial}}^{n}\right\}, \quad g_{\max }^{n}=\max \left\{g_{1}^{n}, \ldots, g_{N_{\partial}}^{n}\right\},
$$

$$
f_{\text {min }}^{n}=\min \left\{0, f_{1}^{(n, \theta)}, \ldots, f_{N_{0}}^{(n, \theta)}\right\}, \quad f_{\max }^{n}=\max \left\{0, f_{1}^{(n, \theta)}, \ldots, f_{N_{0}}^{(n, \theta)}\right\},
$$

$$
\mathbf{e}_{0}=[1, \ldots, 1]^{T} \in \mathbf{R}^{N_{0}}, \mathbf{e}_{\partial}=[1, \ldots, 1]^{T} \in \mathbf{R}^{N_{\partial}}, \mathbf{e}=[1, \ldots, 1]^{T} \in \mathbf{R}^{N} .
$$

We formulate the discrete maximum principle (DMP) for the discrete model (39) as follows:

$$
\begin{aligned}
& \min \left\{0, g_{\text {min }}^{n}, g_{\text {min }}^{n+1}, u_{\text {min }}^{n}\right\}+\Delta t f_{\text {min }}^{n} \leq \\
& \leq u_{i}^{n+1} \leq \max \left\{0, g_{\text {max }}^{n}, g_{\text {max }}^{n+1}, u_{\text {max }}^{n}\right\}+\Delta t f_{\text {max }}^{n},
\end{aligned}
$$

$\left(i=1, \ldots, N_{0} ; n=0,1,2 \ldots\right)$, following [15, p. 100].

In order to satisfy the DMP for the model (39), we also impose conditions for the choice of the time-discretization parameter $\Delta t$ :

$$
A_{i j}=M_{i j}+\theta \Delta t K_{i j} \leq 0 \quad\left(i \neq j, \quad i=1, \ldots, N_{0}, j=1, \ldots, N\right)
$$

(B6) $\quad B_{i i}=M_{i i}-(1-\theta) \Delta t K_{i i} \geq 0 \quad\left(i=1, \ldots, N_{0}\right)$.

The following proposition summarizes some properties of the matrices $\mathbf{A}$ and $\mathbf{B}$.

Lemma 4.1 Under conditions (B1)-(B6) the following properties are valid: 
P1. $\mathbf{A}_{\partial} \leq \mathbf{0}$,

P3. $\mathbf{A}_{0}$ is an invertible matrix and $\mathbf{A}_{0}^{-1} \geq \mathbf{0}$;

P5. $\mathbf{B} \geq \mathbf{0}$;

P7. Ae $\geq \mathrm{Be}$;
P2. $\mathbf{e}_{0} \leq \mathrm{Ae} ;$

P4. $\mathbf{A}_{0}^{-1} \mathbf{A}_{\partial} \leq \mathbf{0}$;

$\mathrm{P} 6 . \mathbf{K e} \geq \mathbf{0}$;

P8. $-\mathbf{A}_{0}^{-1} \mathbf{A}_{\partial} \mathbf{e}_{\partial} \leq \mathbf{e}_{0}$.

Proof. Property P1 follows from assumption (B5). Using assumptions (B2) and (B4), we have

$$
(\mathbf{A e})_{i}=\sum_{j=1}^{N} \mathbf{A}_{i j}=\sum_{j=1}^{N} \mathbf{M}_{i j}+\theta \Delta t \sum_{j=1}^{N} \mathbf{K}_{i j} \geq 1,
$$

which shows the validity of $\mathrm{P} 2$.

Condition B5 implies that $A_{i j} \leq 0$ for all $i \neq j$. Moreover, based on $\mathrm{P} 1$ and $\mathrm{P} 2$, we have the relation

$$
\mathbf{A}_{0} \mathbf{e}_{0} \geq \mathbf{A}_{0} \mathbf{e}_{0}+\mathbf{A}_{\partial} \mathbf{e}_{\partial}=\mathbf{A e} \geq \mathbf{e}_{0}>\mathbf{0} .
$$

Owing to (B5), the off-diagonal elements of $\mathbf{A}_{0}$ are nonpositive. Moreover, there exists a positive vector $\mathbf{e}_{0}>0$ for which $\mathbf{A}_{0} \mathbf{e}_{0}>0$. This yields that $\mathbf{A}_{0}$ is an M-matrix, see e.g. [1, Thm. 2.3]. Hence, the statements P3 and P4 are obvious. Condition (B6) implies that $B_{i i} \geq 0$ for all $i=1,2, \ldots N_{0}$. On the other hand, according to (B1) and (B3), we get $B_{i j} \geq 0$ for all $i \neq j$. Hence, P5 also holds. Property P6 follows from (B2). Using P6, we have

$$
\mathbf{A e}=\mathbf{M e}+\theta \Delta t \mathbf{K e} \geq \mathbf{M e} \geq(\mathbf{M}-(1-\theta) \Delta t \mathbf{K})) \mathbf{e}=\mathbf{B e},
$$

which proves P7. Finally, due to P2 and P1, we have $\mathbf{A}_{0}^{-1} \mathbf{e}_{0} \leq \mathbf{e}_{0}+\mathbf{A}_{0}^{-1} \mathbf{A}_{\partial} \mathbf{e}_{\partial}$. Hence, using P3, we get $-\mathbf{A}_{0}^{-1} \mathbf{A}_{\partial} \mathbf{e}_{\partial} \leq \mathbf{e}_{0}-\mathbf{A}_{0}^{-1} \mathbf{e}_{0} \leq \mathbf{e}_{0}$, which shows the validity of P8. This completes the proof.

Now we can prove the following

Theorem 4.1 Assume that conditions (B1)-(B6) are satisfied. Then the DMP of the form (45) holds for the system (38).

Proof. ¿From (39), using P2, we get

$$
\begin{gathered}
\mathbf{A}_{0} \mathbf{u}^{n+1}+\mathbf{A}_{\partial} \mathbf{g}^{n+1}=\mathbf{A} \overline{\mathbf{u}}^{n+1}=\mathbf{B} \overline{\mathbf{u}}^{n}+\Delta t \mathbf{f}^{(n, \theta)} \leq \\
\leq \mathbf{B} \overline{\mathbf{u}}^{n}+\Delta t f_{\text {max }}^{n} \mathbf{e}_{0} \leq \mathbf{B} \overline{\mathbf{u}}^{n}+\Delta t f_{\text {max }}^{n} \mathbf{A e} .
\end{gathered}
$$

Hence, using P3, and then P5 and P7, respectively, we get

$$
\begin{aligned}
& \mathbf{u}^{n+1} \leq-\mathbf{A}_{0}^{-1} \mathbf{A}_{\partial} \mathbf{g}^{n+1}+\mathbf{A}_{0}^{-1} \mathbf{B} \overline{\mathbf{u}}^{n}+\Delta t f_{\max }^{n} \mathbf{A}_{0}^{-1} \mathbf{A e} \leq \\
& \leq-\mathbf{A}_{0}^{-1} \mathbf{A}_{\partial} \mathbf{g}^{n+1}+v_{\max }^{n} \mathbf{A}_{0}^{-1} \mathbf{B e}+\Delta t f_{\max }^{n} \mathbf{A}_{0}^{-1} \mathbf{A e} \leq \\
& \leq-\mathbf{A}_{0}^{-1} \mathbf{A}_{\partial} \mathbf{g}^{n+1}+v_{\max }^{n} \mathbf{A}_{0}^{-1} \mathbf{A e}+\Delta t f_{\max }^{n} \mathbf{A}_{0}^{-1} \mathbf{A e}= \\
& =-\mathbf{A}_{0}^{-1} \mathbf{A}_{\partial} \mathbf{g}^{n+1}+v_{\max }^{n} \mathbf{A}_{0}^{-1}\left[\mathbf{A}_{0} \mid \mathbf{A}_{\partial}\right] \mathbf{e}+\Delta t f_{\max }^{n} \mathbf{A}_{0}^{-1}\left[\mathbf{A}_{0} \mid \mathbf{A}_{\partial}\right] \mathbf{e}= \\
& =-\mathbf{A}_{0}^{-1} \mathbf{A}_{\partial} \mathbf{g}^{n+1}+v_{\max }^{n}\left(\mathbf{e}_{0}+\mathbf{A}_{0}^{-1} \mathbf{A}_{\partial} \mathbf{e}_{\partial}\right)+ \\
& +\Delta t f_{\max }^{n}\left(\mathbf{e}_{0}+\mathbf{A}_{0}^{-1} \mathbf{A}_{\partial} \mathbf{e}_{\partial}\right) .
\end{aligned}
$$


Regrouping the above inequality, we get

$$
\mathbf{u}^{n+1}-v_{\max }^{n} \mathbf{e}_{0}-\Delta t f_{\max }^{n} \mathbf{e}_{0} \leq-\mathbf{A}_{0}^{-1} \mathbf{A}_{\partial}\left(\mathbf{g}^{n+1}-v_{\max }^{n} \mathbf{e}_{\partial}-\Delta t f_{\max }^{n} \mathbf{e}_{\partial}\right) .
$$

Hence, for the $i$-th coordinate of the both sides of (50), using P4, and finally P8, we obtain

$$
\begin{aligned}
& u_{i}^{n+1}-v_{\text {max }}^{n}-\Delta t f_{\text {max }}^{n} \leq \sum_{j=1}^{N_{\partial}}\left(-\mathbf{A}_{0}^{-1} \mathbf{A}_{\partial}\right)_{i j}\left(g_{j}^{n+1}-v_{\max }^{n}-\Delta t f_{\text {max }}^{n}\right) \leq \\
& \leq\left(\sum_{j=1}^{N_{\partial}}\left(-\mathbf{A}_{0}^{-1} \mathbf{A}_{\partial}\right)_{i j}\right) \cdot \max \left\{0, \max _{j}\left\{g_{j}^{n+1}-v_{\max }^{n}\right\}\right\} \leq \max ^{n}\left\{0, \max _{j}\left\{g_{j}^{n+1}-v_{\max }^{n}\right\}\right\} .
\end{aligned}
$$

$$
\begin{aligned}
& \min \left\{0, g_{\text {min }}^{n}, g_{\text {min }}^{n+1}, u_{\text {min }}^{n}\right\}+\Delta t \min \left\{0, f_{\text {min }}^{n}\right\} \leq \\
& \leq u_{i}^{n+1} \leq \max \left\{0, g_{\text {max }}^{n}, g_{\text {max }}^{n+1}, u_{\text {max }}^{n}\right\}+\Delta t \max \left\{0, f_{\text {max }}^{n}\right\},
\end{aligned}
$$

$\left.=1, \ldots, N_{0} ; n=0,1,2 \ldots\right)$, where

$$
f_{\text {min }}^{n}=\min \left\{f_{1}^{(n, \theta)}, \ldots, f_{N_{0}}^{(n, \theta)}\right\}, \quad f_{\max }^{n}=\max \left\{f_{1}^{(n, \theta)}, \ldots, f_{N_{0}}^{(n, \theta)}\right\} .
$$

\subsection{The general case}

Now we verify that, without loss of generality, we can replace condition (B4) with the less restrictive assumption $\sum_{j=1}^{N} M_{i j}>0$ for all $i$. Further, assumption (B1) can be formally omitted (it will follow from the other ones).

Hence we now impose the following five conditions:

\section{Assumptions 4.3.}

(i) $\sum_{j=1}^{N} K_{i j} \geq 0 \quad$ for all $i=1, \ldots, N_{0}$;

(ii) $M_{i j} \geq 0 \quad$ for all $i=1, \ldots, N_{0}, j=1, \ldots, N$;

(iii) $\sum_{j=1}^{N} M_{i j}=: m_{i}>0 \quad$ for all $i=1, \ldots, N_{0}$;

(iv) $A_{i j}=M_{i j}+\theta \Delta t K_{i j} \leq 0 \quad$ for all $i=1, \ldots, N_{0}, j=1, \ldots, N, i \neq j$;

(v) $\quad B_{i i}=M_{i i}-(1-\theta) \Delta t K_{i i} \geq 0 \quad$ for all $i=1, \ldots, N_{0}$.

Theorem 4.2 Let Assumptions 4.3 hold for the full discretization of the ODE system (35). Then the discrete solution, obtained from (38), satisfies the following DMP:

$$
\begin{aligned}
& \min \left\{0, g_{\text {min }}^{n}, g_{\text {min }}^{n+1}, u_{\text {min }}^{n}\right\}+\Delta t \min \left\{0, \tilde{f}_{\text {min }}^{n}\right\} \leq \\
& \leq u_{i}^{n+1} \leq \max \left\{0, g_{\text {max }}^{n}, g_{\text {max }}^{n+1}, u_{\text {max }}^{n}\right\}+\Delta t \max \left\{0, \tilde{f}_{\text {max }}^{n}\right\},
\end{aligned}
$$


$\left(i=1, \ldots, N_{0} ; n=0,1,2 \ldots\right)$, where, using $m_{i}$ from Assumption 4.3 (iii),

$$
\tilde{f}_{\text {min }}^{n}=\min \left\{\frac{f_{1}^{(n, \theta)}}{m_{1}}, \ldots, \frac{f_{N_{0}}^{(n, \theta)}}{m_{N_{0}}}\right\}, \quad \tilde{f}_{\max }^{n}=\max \left\{\frac{f_{1}^{(n, \theta)}}{m_{1}}, \ldots, \frac{f_{N_{0}}^{(n, \theta)}}{m_{N_{0}}}\right\} .
$$

Proof. Introducing the diagonal matrix $\mathbf{D}=\operatorname{diag}\left[m_{1}, \ldots m_{N_{0}}\right]$, we can rewrite the original equation (35) in the equivalent form

$$
\mathbf{D}^{-1} \mathbf{M} \frac{\mathrm{d} \mathbf{u}}{\mathrm{d} t}+\mathbf{D}^{-1} \mathbf{K u}=\mathbf{D}^{-1} \mathbf{f} .
$$

Assumptions 4.3 (i)-(ii) and (iv)-(v) for the matrices in (35) are equivalent to the properties (B2)-(B3) and (B5)-(B6) for the matrix in (56), and assumption (iii) implies that the matrix $\mathbf{D}^{-1} \mathbf{M}$ satisfies the condition (B4). Finally, assumptions (B3) and (B5) imply that $\theta$ must be positive, in which case assumption (B1) follows from (B5). Consequently, Theorem 4.1 can be applied to system (56). By Remark 4.1, this means that (52) holds such that $\mathbf{f}$ is replaced by $\mathbf{D}^{-1} \mathbf{f}$, i.e. $f_{\min }^{n}$ and $f_{\max }^{n}$ are replaced by $\tilde{f}_{\min }^{n}$ and $\tilde{f}_{\max }^{n}$, respectively.

The above result still reduces the values of $u$ on the $(n+1)$ th time level to the values of $u$ on $n$th time level. Now, by induction, we obtain a DMP that reduces the values of $u$ only to the input data until the $(n+1)$ th time level:

Theorem 4.3 Let Assumptions 4.3 hold and let us introduce notations

$$
\begin{array}{ll}
g_{\min }^{(n)}:=\min \left\{g_{\min }^{0}, \ldots, g_{\min }^{n+1}\right\}, & \hat{f}_{\text {min }}^{(n)}:=\min \left\{\hat{f}_{\min }^{0}, \ldots, \hat{f}_{\min }^{n}\right\}, \\
g_{\text {max }}^{(n)}:=\max \left\{g_{\text {max }}^{0}, \ldots, g_{\text {max }}^{n+1}\right\}, & \hat{f}_{\text {max }}^{(n)}:=\max \left\{\hat{f}_{\max }^{0}, \ldots, \hat{f}_{\max }^{n}\right\} .
\end{array}
$$

Then we have

$$
\min \left\{0, g_{\text {min }}^{(n)}, u_{\text {min }}^{(0)}\right\}+(n+1) \Delta t \min \left\{0, \hat{f}_{\text {min }}^{(n)}\right\} \leq u_{i}^{n+1} \leq \max \left\{0, g_{\text {max }}^{(n)}, u_{\max }^{(0)}\right\}+(n+1) \Delta t \max \left\{0, \hat{f}_{\max }^{(n)}\right\}
$$

Proof. The result follows directly from the previous theorem by mathematical induction.

Of course, the values in (57) can be further estimated by the global minima and maxima of $\mathbf{g}$ and $\mathbf{f}$ for $n=0, \ldots, n_{T}-1$ independently of $n$, which shows the analogy with the continuous case (33).

\section{The discrete maximum principle for the nonlinear system}

\subsection{Reformulation of the problem}

First we rewrite problem (10) to a problem with nonlinear coefficients. Let us define, for any $k, l=1, \ldots, s, x \in \Omega$ resp. $\Gamma, t>0, \xi \in \mathbf{R}^{s}$,

$$
r_{k l}(x, t, \xi):=\int_{0}^{1} \frac{\partial q_{k}}{\partial \xi_{l}}(x, t, \alpha \xi) d \alpha, \quad z_{k l}(x, t, \xi):=\int_{0}^{1} \frac{\partial s_{k}}{\partial \xi_{l}}(x, t, \alpha \xi) d \alpha
$$


and

$$
\hat{f}_{k}(x, t):=f_{k}(x, t)-q_{k}(x, t, 0), \quad \hat{\gamma}_{k}(x, t):=\gamma_{k}(x, t)-s_{k}(x, t, 0) .
$$

Then the Newton-Leibniz formula yields for all $x, t, \xi$ that

$$
q_{k}(x, t, \xi)-q_{k}(x, t, 0)=\sum_{l=1}^{s} r_{k l}(x, t, \xi) \xi_{l}, \quad s_{k}(x, t, \xi)-s_{k}(x, t, 0)=\sum_{l=1}^{s} z_{k l}(x, t, \xi) \xi_{l} .
$$

and $\langle\psi(t), v\rangle:=\int_{\Omega} \sum_{k=1}^{s} \hat{f}_{k} v_{k} d x+\int_{\Gamma} \sum_{k=1}^{s} \hat{\gamma}_{k} v_{k} d \sigma$.

Then the semidiscretization of the problem reads as follows: find a vector function $u_{h}=u_{h}(x, t)$ such that

$u_{k}^{h}(x, 0)=u_{k}^{(0), h}(x) \quad(x \in \Omega), \quad u_{k}^{h}(., t)-g_{k}^{h}(., t) \in V_{0}^{h} \quad(t \in(0, T))$, for all $k=1, \ldots, s$ and

$$
\int_{\Omega} \sum_{k=1}^{s} \frac{\partial u_{k}^{h}}{\partial t} v_{k}^{h} d x+B\left(t, u_{h} ; u_{h}, v^{h}\right)=\left\langle\psi(t), v^{h}\right\rangle \quad\left(\forall v^{h} \in V_{0}^{h}, \quad t \in(0, T)\right) .
$$

Proceeding as in (20)-(26), the Cauchy problem for the system of ordinary differential equations (26) takes the following form:

$$
\mathbf{M} \frac{\mathrm{d} \mathbf{u}^{h}}{\mathrm{~d} t}+\mathbf{K}\left(t, \mathbf{u}^{h}\right) \mathbf{u}^{h}=\hat{\mathbf{f}}(t), \quad \mathbf{u}^{h}(0)=\mathbf{u}_{0}^{h}
$$

where $\mathbf{M}$ is as in (26),

$$
\mathbf{K}\left(t, \mathbf{u}^{h}\right)=\left[K\left(t, \mathbf{u}^{h}\right)_{i j}\right]_{N_{0} \times N}, \quad K\left(t, \mathbf{u}^{h}\right)_{i j}:=B\left(t, u_{h} ; \phi_{j}, \phi_{i}\right)
$$

280

$$
\hat{\mathbf{f}}(t)=\left[\hat{f}_{i}(t)\right]_{i=1, \ldots, N_{0}}, \quad \hat{f}_{i}(t)=\int_{\Omega} \hat{f}_{k_{0}}(x, t) \varphi_{p}(x) d x+\int_{\Gamma} \hat{\gamma}_{k_{0}}(x, t) \varphi_{p}(x) d \sigma(x) .
$$


The full discretization reads as

$$
\mathbf{M} \mathbf{u}^{n+1}+\theta \Delta t \mathbf{K}\left(t_{n+1}, \mathbf{u}^{n+1}\right) \mathbf{u}^{n+1}=\mathbf{M} \mathbf{u}^{n}-(1-\theta) \Delta t \mathbf{K}\left(t_{n}, \mathbf{u}^{n}\right) \mathbf{u}^{n}+\Delta t \hat{\mathbf{f}}^{(n, \theta)} .
$$

Since we have set $\mathbf{G}\left(t, \mathbf{u}^{h}\right)=\mathbf{K}\left(t, \mathbf{u}^{h}\right) \mathbf{u}^{h}$ in (26), the expressions (30)-(31) become

$$
\mathbf{P}\left(\mathbf{u}^{n+1}\right)=\left(\mathbf{M}+\theta \Delta t \mathbf{K}\left(t_{n+1}, \mathbf{u}^{n+1}\right)\right) \mathbf{u}^{n+1}, \quad \mathbf{Q}\left(\mathbf{u}^{n}\right)=\left(\mathbf{M}-(1-\theta) \Delta t \mathbf{K}\left(t_{n}, \mathbf{u}^{n}\right)\right) \mathbf{u}^{n}
$$

respectively. Then, letting

$$
\mathbf{A}\left(\mathbf{u}^{n}\right):=\mathbf{M}+\theta \Delta t \mathbf{K}\left(t_{n}, \mathbf{u}^{n}\right), \quad \mathbf{B}\left(\mathbf{u}^{n}\right):=\mathbf{M}-(1-\theta) \Delta t \mathbf{K}\left(t_{n}, \mathbf{u}^{n}\right) \quad\left(n=0,1,2, \ldots, n_{T}\right),
$$

the iteration procedure (66) takes the form

$$
\mathbf{A}\left(\mathbf{u}^{n+1}\right) \mathbf{u}^{n+1}=\mathbf{B}\left(\mathbf{u}^{n}\right) \mathbf{u}^{n}+\Delta t \hat{\mathbf{f}}^{(n, \theta)},
$$

which is similar to (38), but now the coefficient matrices depend on $\mathbf{u}^{n+1}$ resp. $\mathbf{u}^{n}$.

\subsection{The DMP: problems with sublinear growth}

Let us consider Assumptions 2.1, where we let $p_{1}=p_{2}=2$ in assumption (A5), i.e. we have

Assumption $\left(A 5^{\prime}\right)$ : there exist constants $\alpha_{1}, \alpha_{2} \geq 0$ such that for any $x \in \Omega$ (or $x \in \Gamma$, resp.), $t \in(0, T)$ and $\xi \in \mathbf{R}$, and any $k, l=1, \ldots, s$,

$$
\left|\frac{\partial q_{k}}{\partial \xi_{l}}(x, t, \xi)\right| \leq \alpha_{1}, \quad\left|\frac{\partial s_{k}}{\partial \xi_{l}}(x, t, \xi)\right| \leq \alpha_{2}
$$

In what follows, we will need the standard notion of (patch-)regularity of the considered meshes.

Definition 5.1 Let $\Omega \subset \mathbf{R}^{d}$ and let us consider a family of FEM subspaces $\mathcal{V}=\left\{V_{h}\right\}_{h \rightarrow 0}$. The corresponding family of $\mathrm{FE}$ meshes will be called quasi-regular if there exist constants $c_{0}, c_{1}>0$ and a constant $1 \leq \sigma<2$ such that for any $h>0$ and basis function $\phi_{p}$,

$$
c_{1} h^{\sigma} \leq \operatorname{diam}\left(\operatorname{supp} \phi_{p}\right) \leq c_{0} h \text { and } \operatorname{meas}_{d-1}\left(\partial\left(\operatorname{supp} \phi_{p}\right)\right) \leq c_{2} h^{d-1}
$$

(where supp denotes the support, i.e. the closure of the set where the function does not vanish, and meas $_{d-1}$ denotes $(d-1)$-dimensional measure of the boundary of $\left.\operatorname{supp} \phi_{p}\right)$, further, there exist constants $c_{\text {grad }}>0$ and $1 \leq \varrho \leq \frac{2}{\sigma}$ (independent of the basis functions and $h$ ) such that

$$
\max \left|\nabla \varphi_{p}\right| \leq \frac{c_{\text {grad }}}{\operatorname{diam}\left(\operatorname{supp} \varphi_{p}\right)^{\varrho}} \quad(p=1, \ldots, \bar{n}) .
$$

Note that the first inequality in (70) implies

$$
\operatorname{meas}_{d}\left(\operatorname{supp} \phi_{p}\right) \leq c_{3} h^{d}
$$

and in fact it also implies the second inequality in (70) under certain natural but additional assumptions, e.g. if $\operatorname{supp} \phi_{p}$ are convex, as is usually the case for linear, bilinear or prismatic elements. 
Theorem 5.1 Let problem (1)-(5) satisfy Assumptions 2.1 such that we let $p_{1}=p_{2}=2$ in (7), i.e. (A5) reduces to assumption (A5') above. Let us consider a family of finite element subspaces $\mathcal{V}=\left\{V_{h}\right\}_{h \rightarrow 0}$ such that the basis functions satisfy (13)-(14), and the family of associated FE meshes is quasi-regular as in Definition 5.1. Let the following assumptions hold:

(i) for any $p=1, \ldots, n_{0}, q=1, \ldots, n(p \neq q)$, if meas $_{d}\left(\operatorname{supp} \varphi_{p} \cap \operatorname{supp} \varphi_{q}\right)>0$ then

$$
\nabla \varphi_{p} \cdot \nabla \varphi_{q} \leq 0 \quad \text { on } \Omega \quad \text { and } \quad \int_{\Omega} \nabla \varphi_{p} \cdot \nabla \varphi_{q} \leq-K_{0} h^{d-2}
$$

with some constant $K_{0}>0$ independent of $p, q$ and $h$;

(ii) the mesh parameter $h$ satisfies $h<h_{0}$, where $h_{0}>0$ is the first positive root of the equation

$$
-\frac{\mu_{0} K_{0}}{c_{3}} \frac{1}{h^{2}}+\alpha_{1}+\frac{\omega}{c_{3} h^{\varrho \sigma}}=0
$$

where, using notation $\|\mathbf{w}\|_{\infty}:=\sup _{k, x, t}\left|\mathbf{w}_{k}(x, t)\right|$,

$$
\omega:=c_{2} \alpha_{2}+c_{\text {grad }}\|\mathbf{w}\|_{\infty} ;
$$

(iii) using $\omega$ from (74), we have

$$
\Delta t \geq \frac{c_{3} h^{2}}{\theta\left(\mu_{0} K_{0}-\alpha_{1} c_{3} h^{2}-\omega h^{2-\varrho \sigma}\right)}
$$

(iv) if $\theta<1$ then

$$
\Delta t \leq \frac{1}{(1-\theta) R(h)},
$$

using the notations

$$
R(h):=\left(\mu_{1}+\frac{\|\mathbf{w}\|_{\infty}}{2}\right) N(h)+\alpha_{2} G(h)+\left(\alpha_{1}+\frac{\|\mathbf{w}\|_{\infty}}{2}\right),
$$

$$
N(h):=\max _{p=1, \ldots, \bar{n}_{0}} \frac{\int_{\Omega}\left|\nabla \varphi_{p}\right|^{2}}{\int_{\Omega} \varphi_{p}^{2}}, \quad G(h):=\max _{p=1, \ldots, \bar{n}_{0}} \frac{\int_{\Gamma_{N}} \varphi_{p}^{2}}{\int_{\Omega} \varphi_{p}^{2}} .
$$

Then the matrices $\mathbf{M}, \mathbf{K}\left(t_{n+1}, \mathbf{u}^{n+1}\right), \mathbf{A}\left(\mathbf{u}^{n+1}\right)$ and $\mathbf{B}\left(\mathbf{u}^{n}\right)$, defined via (25), (64) and (67)-(68), respectively, have the following properties:

(1) $\sum_{j=1}^{N} \mathbf{K}\left(t_{n+1}, \mathbf{u}^{n+1}\right)_{i j} \geq 0 \quad$ for all $i=1, \ldots, N_{0}$;

(2) $\mathbf{M}_{i j} \geq 0 \quad$ for all $i=1, \ldots, N_{0}, j=1, \ldots, N$;

(3) $\sum_{j=1}^{N} \mathbf{M}_{i j}=: m_{i}>0 \quad$ for all $i=1, \ldots, N_{0}$; 
(4) $\mathbf{A}\left(\mathbf{u}^{n+1}\right)_{i j} \leq 0 \quad\left(i \neq j, \quad i=1, \ldots, N_{0}, j=1, \ldots, N\right)$;

(5) $\mathbf{B}\left(\mathbf{u}^{n}\right)_{i i} \geq 0 \quad\left(i=1, \ldots, N_{0}\right)$.

Proof. First we calculate $K\left(t, \mathbf{u}^{h}\right)_{i j}:=B\left(t, u_{h} ; \phi_{j}, \phi_{i}\right)$ for given $i=1, \ldots, N_{0}, j=$ $1, \ldots, N$. Let us write the indices $i, j$ in the form as in $(22)$ :

$$
i=\left(k_{0}-1\right) \bar{n}_{0}+p \quad \text { for some } 1 \leq k_{0} \leq s \text { and } 1 \leq p \leq \bar{n}_{0},
$$

$$
\begin{gathered}
j=\left(l_{0}-1\right) \bar{n}_{0}+q \quad \text { for some } 1 \leq l_{0} \leq s \text { and } 1 \leq q \leq \bar{n}_{0} \text { or } \\
j=N_{0}+\left(l_{0}-1\right)\left(\bar{n}-\bar{n}_{0}\right)+q-\bar{n}_{0} \quad \text { for some } 1 \leq l_{0} \leq s \text { and } \bar{n}_{0}+1 \leq q \leq \bar{n} .
\end{gathered}
$$

Then the functions $u=\phi_{j}$ and $v=\phi_{i}$ have $l$ th and $k$ th coordinates $u_{l}=\delta_{l, l_{0}} \varphi_{q}$ and $v_{k}=\delta_{k, k_{0}} \varphi_{p}$ (where $\delta_{\text {.,. }}$ is the Kronecker symbol) for $k, l=1, \ldots, s$, hence by (62),

$$
K\left(t, \mathbf{u}^{h}\right)_{i j}=\left\{\begin{aligned}
& \int_{\Omega} r_{k_{0} l_{0}}\left(x, t, u_{h}\right) \varphi_{p} \varphi_{q} d x+\int_{\Gamma} z_{k_{0} l_{0}}\left(x, t, u_{h}\right) \varphi_{p} \varphi_{q} d \sigma \quad \text { if } k_{0} \neq l_{0} ; \\
\int_{\Omega}\left(a_{k_{0}}\left(x, t, u_{h}, \nabla u_{h}\right) \nabla \varphi_{p} \cdot \nabla \varphi_{q}+\left(\mathbf{w}_{k_{0}}(x, t) \cdot \nabla \varphi_{p}\right) \varphi_{q}+r_{k_{0} k_{0}}\left(x, t, u_{h}\right) \varphi_{p} \varphi_{q}\right) d x & \\
& +\int_{\Gamma} z_{k_{0} k_{0}}\left(x, t, u_{h}\right) \varphi_{p} \varphi_{q} d \sigma \quad \text { if } k_{0}=l_{0} .
\end{aligned}\right.
$$

Similarly,

$$
M_{i j}=0 \quad \text { if } k_{0} \neq l_{0}, \quad \text { and } \quad M_{i j}=\int_{\Omega} \varphi_{p} \varphi_{q} d x \quad \text { if } k_{0}=l_{0} .
$$

Now we can prove the desired properties (1)-(5). Moreover, we prove them in general for all $t$ and $\mathbf{u}^{h}$ (but will use them later only in the case formulated in the theorem).

(1) Let $i \in\left\{1, \ldots, N_{0}\right\}$ be fixed. Then, using the notations of (22),

$$
\begin{aligned}
\sum_{j=1}^{N} K\left(t, \mathbf{u}^{h}\right)_{i j}= & \int_{\Omega}\left(a_{k_{0}}\left(x, t, u_{h}, \nabla u_{h}\right) \nabla \varphi_{p} \cdot \nabla\left(\sum_{q=1}^{\bar{n}} \varphi_{q}\right)+\left(\mathbf{w}_{k_{0}}(x, t) \cdot \nabla \varphi_{p}\right)\left(\sum_{q=1}^{\bar{n}} \varphi_{q}\right)\right. \\
& \left.+\left(\sum_{l_{0}=1}^{s} r_{k_{0} l_{0}}\left(x, t, u_{h}\right)\right) \varphi_{p}\left(\sum_{q=1}^{\bar{n}} \varphi_{q}\right)\right) d x+\int_{\Gamma}\left(\sum_{l_{0}=1}^{s} z_{k_{0}} l_{0}\left(x, t, u_{h}\right)\right) \varphi_{p}\left(\sum_{q=1}^{\bar{n}} \varphi_{q}\right) d \sigma .
\end{aligned}
$$

We now use (13) and first estimate the last terms. Using (59), the sums of functions $r_{k l}$ and $z_{k l}$ inherit the nonnegativity (9), hence from (13) we altogether obtain that the last two integrands are nonnegative. Then, (13) also yields that the first integrand vanishes and the sum in the second integrand equals 1 , thus we obtain

$$
\sum_{j=1}^{N} K\left(t, \mathbf{u}^{h}\right)_{i j} \geq \int_{\Omega} \mathbf{w}_{k_{0}}(x, t) \cdot \nabla \varphi_{p}
$$


For fixed $t$, using the divergence theorem and Assumption 2.1 (A4),

332

$$
K\left(t, \mathbf{u}^{h}\right)_{i j} \geq \int_{\Omega}\left(\mathbf{w}_{k_{0}}(x, t) \cdot \nabla \varphi_{p}\right)=\int_{\Gamma_{N}}\left(\mathbf{w}_{k_{0}}(x, t) \cdot \nu\right) \varphi_{p} d \sigma+\int_{\Gamma_{\text {int }}}\left[\mathbf{w}_{k_{0}}(x, t) \cdot \nu\right] \varphi_{p} d \sigma
$$

$$
-\int_{\Omega}\left(\operatorname{div} \mathbf{w}_{k_{0}}(x, t)\right) \varphi_{p} d x \geq 0 .
$$

333

334

since $\sum_{j=1}^{N} M_{i j}=\int_{\Omega} \varphi_{p}\left(\sum_{q=1}^{\bar{n}} \varphi_{q}\right)=\int_{\Omega} \varphi_{p}>0$.

(4) We calculate $A\left(t, \mathbf{u}^{h}\right)_{i j}:=\mathbf{M}_{i j}+\theta \Delta t \mathbf{K}\left(t, \mathbf{u}^{h}\right)_{i j}$ and check its nonpositivity for all $t$ and $\mathbf{u}^{h}$. If $k_{0} \neq l_{0}$ then

$$
\mathbf{A}\left(t, \mathbf{u}^{h}\right)_{i j}=\theta \Delta t\left(\int_{\Omega} r_{k_{0} l_{0}}\left(x, t, u_{h}\right) \varphi_{p} \varphi_{q} d x+\int_{\Gamma} z_{k_{0} l_{0}}\left(x, t, u_{h}\right) \varphi_{p} \varphi_{q} d \sigma\right) \leq 0,
$$

336

using (13) and that by (59), $r_{k_{0} l_{0}}$ and $z_{k_{0} l_{0}}$ inherit the nonpositivity (8).

If $k_{0}=l_{0}$ then

$$
\begin{aligned}
\mathbf{A}\left(t, \mathbf{u}^{h}\right)_{i j}=\int_{\Omega} \varphi_{p} \varphi_{q} d x & +\theta \Delta t \int_{\Omega}\left(a_{k_{0}}\left(x, t, u_{h}, \nabla u_{h}\right) \nabla \varphi_{p} \cdot \nabla \varphi_{q}+\left(\mathbf{w}_{k_{0}}(x, t) \cdot \nabla \varphi_{p}\right) \varphi_{q}\right. \\
& \left.+r_{k_{0} k_{0}}\left(x, t, u_{h}\right) \varphi_{p} \varphi_{q}\right) d x+\theta \Delta t \int_{\Gamma} z_{k_{0} k_{0}}\left(x, t, u_{h}\right) \varphi_{p} \varphi_{q} d \sigma .
\end{aligned}
$$

337

Let $\Omega_{p q}:=\operatorname{supp} \varphi_{p} \cap \operatorname{supp} \varphi_{q}$. Here (13) and (72) yield

$$
\int_{\Omega} \varphi_{p} \varphi_{q} \leq \operatorname{meas}_{d}\left(\Omega_{p q}\right) \leq c_{3} h^{d}
$$

and similarly, also using (70),

$$
\int_{\Omega} r_{k_{0} k_{0}}\left(x, t, u_{h}\right) \varphi_{p} \varphi_{q} \leq \alpha_{1} c_{3} h^{d}, \quad \int_{\Gamma} z_{k_{0} k_{0}}\left(x, t, u_{h}\right) \varphi_{p} \varphi_{q} \leq \alpha_{2} c_{2} h^{d-1}
$$

339

$$
\int_{\Omega} a_{k_{0}}\left(x, t, u_{h}, \nabla u_{h}\right) \nabla \varphi_{p} \cdot \nabla \varphi_{q} \leq-\mu_{0} K_{0} h^{d-2}, \quad \int_{\Omega}\left(\mathbf{w}_{k_{0}}(x, t) \cdot \nabla \varphi_{p}\right) \varphi_{q} \leq c_{g r a d}\|\mathbf{w}\|_{\infty} h^{d-\varrho \sigma} .
$$


Altogether, we obtain

$$
\mathbf{A}\left(t, \mathbf{u}^{h}\right)_{i j} \leq c_{3} h^{d}\left[1+\theta \Delta t\left(-\frac{\mu_{0} K_{0}}{c_{3}} \frac{1}{h^{2}}+\alpha_{1}+\frac{c_{2} \alpha_{2}+c_{g r a d}\|\mathbf{w}\|_{L^{\infty}(\Omega)^{s}}}{c_{3} h^{\varrho \sigma}}\right)\right] .
$$

Since $\varrho \sigma<2$ and $h<h_{0}$ for $h_{0}$ defined in assumption (ii), it follows that we have a negative coefficient of $\theta \Delta t$ above, and from (74) and (75) we obtain that the expression in the large brackets is nonpositive, hence $\mathbf{A}\left(t, \mathbf{u}^{h}\right)_{i j} \leq 0$.

(5) We have $B\left(t, \mathbf{u}^{h}\right)_{i i}:=M_{i i}-(1-\theta) \Delta t K\left(t, \mathbf{u}^{h}\right)_{i i} \geq 0$ iff

$$
\begin{aligned}
\int_{\Omega} \varphi_{p}^{2} \geq(1-\theta) \Delta t\left[\int_{\Omega}(\right. & a_{k_{0}}\left(x, t, u_{h}, \nabla u_{h}\right)\left|\nabla \varphi_{p}\right|^{2}+\left(\mathbf{w}_{k_{0}}(x, t) \cdot \nabla \varphi_{p}\right) \varphi_{p} \\
& \left.\left.+r_{k_{0} k_{0}}\left(x, t, u_{h}\right) \varphi_{p}^{2}\right) d x+\int_{\Gamma} z_{k_{0} k_{0}}\left(x, t, u_{h}\right) \varphi_{p}^{2} d \sigma\right] .
\end{aligned}
$$

The latter holds for all $\Delta t>0$ if $\theta=1$ (i.e. the scheme is implicit). If $\theta<1$, then we estimate the expresssion in brackets from above by

$$
\begin{aligned}
& \int_{\Omega}\left(\mu_{1}\left|\nabla \varphi_{p}\right|^{2}+\|\mathbf{w}\|_{\infty}\left|\nabla \varphi_{p}\right| \varphi_{p}+\alpha_{1} \varphi_{p}^{2}\right)+\int_{\Gamma} \alpha_{2} \varphi_{p}^{2} \\
& \quad \leq \int_{\Omega}\left(\left(\mu_{1}+\frac{\|\mathbf{w}\|_{\infty}}{2}\right)\left|\nabla \varphi_{p}\right|^{2}+\left(\alpha_{1}+\frac{\|\mathbf{w}\|_{\infty}}{2}\right) \varphi_{p}^{2}\right)+\int_{\Gamma} \alpha_{2} \varphi_{p}^{2} \leq R(h) \cdot \int_{\Omega} \varphi_{p}^{2},
\end{aligned}
$$

which shows that (88) holds for all $\Delta t$ that satisfies (76).

Remark 5.1 (Discussion of the assumptions in Theorem 5.1.) We may state similar comments as in the scalar case [13]:

(i) Assumption (i) can be ensured by suitable geometric properties of the space mesh, see subsection 5.4 below.

(ii) The value of $h_{0}$ can be computed easily since it is defined by an equation containing given or computable constants from the assumptions on the coefficients, the mesh quasiregularity and geometry.

(iii) It is well-known from the above works on linear parabolic equations that the usual requirement for the relation between the space and time discretization steps is generally to keep their ratio between two positive constants as they tend to 0, i.e.

$$
\Delta t=O\left(h^{2}\right)
$$

should hold, in order both to achieve convergence in the maximum norm and to satisfy the DMP $[9,10,32]$. We obtain similar properties in Theorem 5.1 for our nonlinear systems. Namely, first, the lower bound in (75) is asymptotically of the form $\Delta t \geq O\left(h^{2}\right)$ as $h \rightarrow 0$, and all the constants involved are easily computable. If $\theta=1$, i.e. the scheme is implicit, then there is no upper restriction on $\Delta t$. If $\theta<1$, then for various popular finite elements one has $R(h)=O\left(h^{-2}\right)$ in (77), see [13]. (Namely, this has been proved so far for 
simplicial elements in any dimension, bilinear elements in 2D and prismatic elements in 3D.) Hence $\Delta t \leq O\left(h^{2}\right)$ as $h \rightarrow 0$, which yields with the other bound the usual condition (89) (as $h \rightarrow 0$ ) for the space and time discretizations.

In addition, the lower bound in (75) must be smaller than the upper bound in (76). In view of the factor $1-\theta$ in the latter, this gives a restriction on $\theta$ to be close enough to 1 , similarly to the linear case.

Now we can derive the corresponding discrete maximum principles. First, based on Theorem 4.2, we obtain

Corollary 5.1 Let problem (1)-(5) and its FE discretization satisfy the conditions of Theorem 5.1. Then the discrete solution, obtained from (68), satisfies the discrete maximum principles (54) and (58).

One is more interested in the information containing the original coefficients rather than the discrete values in (54). In this respect we can derive the following result:

Lemma 5.1 Let problem (1)-(5) and its FE discretization satisfy the conditions of Theorem 5.1.

If the functions $u_{k}^{(0)}, g_{k}$ and $f_{k}$ are also continuous on the closure of their domains, then the discrete solution, obtained from (68), satisfies the following discrete maximum principle:

$$
\begin{aligned}
& u_{i}^{n+1} \leq \max \left\{0, \max _{k=1, \ldots, s} \max _{\bar{\Gamma}_{(n+1) \Delta t}^{D}} g_{k}^{h}, \max _{k=1, \ldots, s} \max _{\bar{\Omega}} u_{k}^{(0), h}\right\} \\
& \quad+(n+1) \Delta t \max \left\{0, \max _{k=1, \ldots, s} \max _{\bar{Q}_{(n+1) \Delta t}} \hat{f}_{k}+D(h) \max _{k=1, \ldots, s} \max _{\bar{\Gamma}_{(n+1) \Delta t}} \hat{\gamma}_{k}\right\}
\end{aligned}
$$

where $\Gamma_{(n+1) \Delta t}^{D}:=\Gamma_{D} \times[0,(n+1) \Delta t], \quad \Gamma_{(n+1) \Delta t}:=\Gamma \times[0,(n+1) \Delta t], \quad Q_{(n+1) \Delta t}:=$ $\Omega \times[0,(n+1) \Delta t]$, further, from (60),

$$
\hat{f}_{k}(x, t):=f_{k}(x, t)-q_{k}(x, t, 0), \quad \hat{\gamma}_{k}(x, t):=\gamma_{k}(x, t)-s_{k}(x, t, 0)
$$

and finally, $D(h):=\max _{p=1, \ldots, \bar{n}} \frac{\int_{\Gamma_{N}} \varphi_{p} d \sigma}{\int_{\Omega} \varphi_{p} d x}$.

The reverse of the above inequality (discrete minimum principle) holds if all maxima are replaced by minima.

If we do not assume $u_{k}^{(0)}, g_{k}$ and $f_{k}$ to be continuous on the closure of their domains, then the above inequalities hold if the corresponding max and min are replaced by ess sup and ess inf.

Proof. We only prove the first, major, statement. (The other two are then obvious.) In view of Corollary 5.1, we must estimate further the r.h.s. of (58):

$$
u_{i}^{n+1} \leq \max \left\{0, g_{\max }^{(n)}, u_{\max }^{(0)}\right\}+(n+1) \Delta t \max \left\{0, \hat{f}_{\max }^{(n)}\right\} .
$$


Using the definitions, we first have

$$
\begin{aligned}
& g_{\text {max }}^{(n)}=\max \left\{g_{p}^{(k)}(j \Delta t): j=0, \ldots, n+1, k=1, \ldots, s, p=1, \ldots, \bar{n}_{\partial}\right\} \\
& \quad \leq \max \left\{g_{p}^{(k)}(t): 0 \leq t \leq(n+1) \Delta t, k=1, \ldots, s, p=1, \ldots, \bar{n}_{\partial}\right\} .
\end{aligned}
$$

Here (14) and (19) imply $g_{p}^{(k)}(t)=g_{k}\left(B_{\bar{n}_{0}+p}, t\right)$, hence $g_{\max }^{(n)} \leq \max \left\{g_{k}(x, t): x \in \bar{\Gamma}_{D}, 0 \leq\right.$ $t \leq(n+1) \Delta t, \quad k=1, \ldots, s\}=\max _{k=1, \ldots, s, s} \max _{\bar{\Gamma}_{(n+1) \Delta t}^{D}} g_{k}^{h}$. Second, we similarly obtain

$$
\begin{gathered}
u_{\text {max }}^{(0)}=\max \left\{u_{p}^{(k)}(0): k=1, \ldots, s, p=1, \ldots, \bar{n}_{\partial}\right\}=\max \left\{u_{k}^{(0)}\left(B_{p}\right): k=1, \ldots, s, p=1, \ldots, \bar{n}_{\partial}\right\} \\
\leq \max \left\{u_{k}^{(0)}(x): x \in \bar{\Omega}, k=1, \ldots, s\right\}=\max _{k=1, \ldots, s} \max _{\bar{\Omega}} u_{k}^{(0), h} .
\end{gathered}
$$

Finally, from (28), (55) and (65) we have

$$
\begin{gathered}
\hat{f}_{\text {max }}^{(n)}=\max _{i=1, \ldots, N} \frac{1}{m_{i}}\left(\theta \hat{f}_{i}((n+1) \Delta t)+(1-\theta) \hat{f}_{i}(n \Delta t)\right) \\
=\max _{i=1, \ldots, N} \frac{1}{m_{i}}\left(\int_{\Omega}\left(\theta \hat{f}_{k_{0}}(x,(n+1) \Delta t)+(1-\theta) \hat{f}_{k_{0}}(x, n \Delta t)\right) \varphi_{p} d x\right. \\
\left.+\int_{\Gamma}\left(\theta \hat{\gamma}_{k_{0}}(x,(n+1) \Delta t)+(1-\theta) \gamma_{k_{0}}(x, n \Delta t)\right) \varphi_{p} d \sigma\right) .
\end{gathered}
$$

By definition and (84),

$$
\begin{gathered}
\hat{f}_{\text {max }}^{(n)} \leq \max _{p=1, \ldots, \bar{n}} \frac{1}{\int_{\Omega} \varphi_{p}}\left(\left(\max _{k=1, \ldots, s} \max _{\bar{Q}_{(n+1) \Delta t}} \hat{f}_{k}\right) \int_{\Omega} \varphi_{p}+\left(\max _{k=1, \ldots, s} \max _{\Gamma_{(n+1) \text { Deltat }}} \hat{\gamma}_{k}\right) \int_{\Gamma} \varphi_{p}\right) \\
\leq \max _{k=1, \ldots, s} \max _{\bar{Q}_{(n+1) \Delta t}} \hat{f}_{k}+D(h) \max _{k=1, \ldots, s} \max _{\bar{\Gamma}_{(n+1) \Delta t}} \hat{\gamma}_{k} .
\end{gathered}
$$

In practical situations the terms with $D(h)$ usually vanish. Namely, one often has $\hat{\gamma}_{k} \equiv 0$ (namely, $\gamma_{k} \equiv 0$ and $s_{k}(x, t, 0) \equiv 0$, e.g. for reaction-diffusion problems), in which case the term containing $\max \hat{\gamma}_{k}$ disappears, and Lemma 5.1 becomes completely analogous to (33). The same holds if there is only Dirichlet boundary. More generally, if the $\hat{\gamma}_{k}$ do not vanish but have a common sign condition, then we have a one-sided analogy. These are summarized as follows:

Theorem 5.2 Let problem (1)-(5) and its FE discretization satisfy the conditions of Theorem 5.1.

If the functions $u_{k}^{(0)}, g_{k}$ and $f_{k}$ are also continuous on the closure of their domains, then the discrete solution, obtained from (68), satisfies the following inequalities, where the notations of Lemma 5.1 are used:

(1) If $\hat{\gamma}_{k} \leq 0$ for all $k=1, \ldots, s$, then

$u_{i}^{n+1} \leq \max \left\{0, \max _{k=1, \ldots, s} \max _{\Gamma_{(n+1) \Delta t}^{D}} g_{k}^{h}, \max _{k=1, \ldots, s} \max _{\bar{\Omega}} u_{k}^{(0), h}\right\}+(n+1) \Delta t \max \left\{0, \max _{k=1, \ldots, s} \max _{\bar{Q}_{(n+1) \Delta t}} \hat{f}_{k}\right\}$. 
(2) If $\hat{\gamma}_{k} \geq 0$ for all $k=1, \ldots, s$, then

$u_{i}^{n+1} \geq \min \left\{0, \min _{k=1, \ldots, s} \min _{\Gamma_{(n+1) \Delta t}^{D}} g_{k}^{h}, \min _{k=1, \ldots, s} \min _{\bar{\Omega}} u_{k}^{(0), h}\right\}+(n+1) \Delta t \min \left\{0, \min _{k=1, \ldots, s} \min _{\bar{Q}_{(n+1) \Delta t}} \hat{f}_{k}\right\}$.

(3) If $\hat{\gamma}_{k} \equiv 0$ for all $k=1, \ldots, s$, or $\Gamma_{N} \cup \Gamma_{\text {int }}=\emptyset$, then both of the above inequalities are valid.

If we do not assume $u_{k}^{(0)}, g_{k}$ and $f_{k}$ to be continuous on the closure of their domains, then the above inequalities hold if the corresponding max and min are replaced by ess sup and ess inf. Finally, $n \Delta t$ can be further bounded uniformly by $T$ in all the estimates.

PROOF. It readily follows from Lemma 5.1.

Finally, using statement (2) above, one can readily derive the frequently relevant discrete nonnegativity principle:

Corollary 5.2 Let problem (1)-(5) and its FE discretization satisfy the conditions of Theorem 5.1.

If $\hat{f}_{k} \geq 0, g_{k}^{h} \geq 0, \hat{\gamma}_{k} \geq 0$ and $u_{k}^{(0), h} \geq 0$ for all $k=1, \ldots, s$, then the fully discrete solution, obtained from (68), satisfies

$$
u_{i}^{n} \geq 0 \quad\left(n=0,1, \ldots, n_{T}, i=1, \ldots, N_{0}\right) .
$$

Remark 5.2 Corollary 5.2 means that the coordinates $u_{k}^{h}$ of the semidiscrete solution are nonnegative in each node point. Properties (13)-(14) of the basis functions imply that the coordinates $u^{h}(., n \Delta t)$ of the FEM solution for all time levels $n \Delta t$ are also nonnegative. If, in addition, we extend the solutions to $Q_{T}$ with values between those on the neighbouring time levels, e.g. with the method of lines, then we obtain that the coordinates of the discrete solution satisfy

$$
u_{k}^{h} \geq 0 \quad \text { on } Q_{T} \quad(k=1, \ldots, s)
$$

\subsection{The DMP: problems with superlinear growth}

In this subsection we allow stronger growth (of power order) of the nonlinearities $q_{k}$ and $s_{k}$ than in the above, i.e. we return to Assumption 2.1 (A5), and extend our DMP results from the previous section to this case. For this we need some extra technical assumptions and results. The discussion of this modification is similar to the scalar case [13], and we may rely on many of the technical results therein.

Let us first summarize the additional conditions.

\section{Assumptions 5.3.}

(B1) We restrict ourselves to the case of implicit scheme: $\theta=1$.

(B2) The coefficients on $\Gamma_{N}$ satisfy $\hat{\gamma}_{k}(x, t):=\gamma_{k}(x, t)-s_{k}(x, t, 0) \equiv 0$ for all $k=1, \ldots, s$, further, $\Gamma_{D} \neq \emptyset$. 
(B3) The coordinates of the exact solution satisfy $u_{k}(., t) \in W^{1, q}(\Omega)$ for some $q>2$ (if $d=2$ ) or some $q \geq 2 d /\left(d-(d-2)\left(p_{1}-2\right)\right.$ ) (if $d \geq 3$ ) for all $t \in[0, T]$.

(B4) The discretization satisfies $M_{p_{1}}:=\sup _{t \in[0, T]}\left\|u(., t)-u_{h}(., t)\right\|_{L^{p_{1}(\Omega)}}<\infty$, further, if $\beta_{2} \neq 0$ in $(7)$ then $M_{p_{2}}:=\sup _{t \in[0, T]}\left\|u_{h}(., t)\right\|_{L^{p_{2}\left(\Gamma_{N}\right)}}<\infty$.

(B5) The diagonal row-dominance (9) is completed with diagonal dominance w.r.t. columns: for all $k=1, \ldots, s, x \in \Omega$ (or $x \in \Gamma_{N} \cup \Gamma_{\text {int }}$, resp.), $t \in(0, T), \xi \in \mathbf{R}^{s}$,

$$
\sum_{l=1}^{s} \frac{\partial q_{l}}{\partial \xi_{k}}(x, t, \xi) \geq 0 . \quad \sum_{l=1}^{s} \frac{\partial s_{l}}{\partial \xi_{k}}(x, t, \xi) \geq 0 .
$$

The full discretization (66) for $\theta=1$ reads as

$$
\mathbf{M} \mathbf{u}^{n+1}+\Delta t \mathbf{K}\left(t_{n+1}, \mathbf{u}^{n+1}\right) \mathbf{u}^{n+1}=\mathbf{M} \mathbf{u}^{n}+\Delta t \hat{\mathbf{f}}^{(n)} .
$$

Let $u^{n+1} \in V_{h}$ denote the function with coefficient vector $\mathbf{u}^{n+1}$, and let $\hat{f}^{n}(x):=\hat{f}(x, n \Delta t)$. Then, by the definition of the mass and stiffness matrices, (91) implies

$$
\int_{\Omega} \sum_{k=1}^{s} u_{k}^{n+1} v_{k} d x+\Delta t B\left(t_{n+1}, u^{n+1} ; u^{n+1}, v\right)=\int_{\Omega} \sum_{k=1}^{s} u_{k}^{n} v_{k} d x+\Delta t\left\langle\psi^{n}, v\right\rangle
$$

(for all $v \in V_{h}$ ), where $\left\langle\psi^{n}, v\right\rangle=\int_{\Omega} \sum_{k=1}^{s} \hat{f}_{k}^{n} v_{k} d x+\int_{\Gamma_{N}} \sum_{k=1}^{s} \hat{\gamma}_{k}^{n} v_{k} d \sigma$. Here, by assumption (B2), the integral on $\Gamma_{N}$ vanishes, further, $\hat{f} \in L^{\infty}\left(Q_{T}\right)$ by Assumption 2.1 (A2).

Then the following technical results hold.

Lemma 5.2 Let Assumptions 5.3 hold. Then

(1) the norms $\left\|u^{n}\right\|_{L^{2}(\Omega)}$ are bounded independently of $n$ and $V_{h}$ by some constant $K_{L_{2}}>0$.

(2) the norms $\left\|u^{n}\right\|_{L^{p_{1}}(\Omega)}$ are bounded independently of $n$ and $V_{h}$ by some constant $K_{p_{1}, \Omega}>0$.

PROOF. It goes in the same way as in Lemmata 5.2-5.3 in [13], if those proofs are now applied to the coordinate functions of the solution. The additional coercive nonsymmetric terms in the equations do not change the derivation in which the bilinear form is dropped due to coercivity. Any of the equivalent finite-dimensional norms can be chosen for the vector function $u^{n}$ using the $L^{2}$ resp. $L^{p_{1}}$ norms of its coordinate functions.

Now we can prove the main result on the discretization matrices:

Theorem 5.3 Let problem (1)-(5) satisfy Assumptions 2.1 and Assumptions 5.3. Let us consider a family of finite element subspaces $\mathcal{V}=\left\{V_{h}\right\}_{h \rightarrow 0}$ such that the basis functions satisfy (13)-(14), and the family of associated FE meshes is quasi-regular as in Definition 5.1. Let the following assumptions hold: 
(i) for any $p=1, \ldots, n_{0}, q=1, \ldots, n(p \neq q)$, if meas $_{d}\left(\operatorname{supp} \varphi_{p} \cap \operatorname{supp} \varphi_{q}\right)>0$ then

$$
\nabla \varphi_{p} \cdot \nabla \varphi_{q} \leq 0 \quad \text { on } \Omega \quad \text { and } \quad \int_{\Omega} \nabla \varphi_{p} \cdot \nabla \varphi_{q} \leq-K_{0} h^{d-2}
$$

with some constant $K_{0}>0$ independent of $p, q$ and $h$;

(ii) the mesh parameter $h$ satisfies $h<h_{0}$, where $h_{0}>0$ is the first positive root of the equation

$$
-\frac{\mu_{0} K_{0}}{c_{3}} \frac{1}{h^{2}}+\alpha_{1}+\frac{\omega}{c_{3} h^{\varrho \sigma}}+\frac{\beta_{1} c_{3}^{\frac{2-p_{1}}{p_{1}}} K_{p_{1}, \Omega}^{p_{1}-2}}{h^{\gamma_{1}}}+\frac{\beta_{2} c_{2}^{\frac{2}{p_{2}}} M_{p_{2}}^{p_{2}-2}}{c_{3} h^{\gamma_{2}}}=0,
$$

where the numbers $0<\gamma_{1}, \gamma_{2}<2$ are defined below in (96), (97), respectively, and $\omega:=c_{2} \alpha_{2}+c_{\text {grad }}\|\mathbf{w}\|_{\infty}$ as in (74);

(iii) we have

$$
\Delta t \geq \frac{c_{3} h^{2}}{\theta\left(\mu_{0} K_{0}-\alpha_{1} c_{3} h^{2}-\omega h^{2-\varrho \sigma}-\beta_{1} c_{3}^{\frac{2}{p_{1}}} K_{p_{1}, \Omega}^{p_{1}-2} h^{2-\gamma_{1}}-\beta_{2} c_{2}^{\frac{2}{p_{2}}} M_{p_{2}}^{p_{2}-2} h^{2-\gamma_{2}}\right)} .
$$

Then the matrices $\mathbf{M}, \mathbf{K}\left(\mathbf{u}^{n+1}\right), \mathbf{A}\left(\mathbf{u}^{n+1}\right)$ and $\mathbf{B}\left(\mathbf{u}^{n}\right)$, defined via (25), (64) and (67)-(68), respectively, have the following properties:

(1) $\sum_{j=1}^{N} K\left(\mathbf{u}^{n+1}\right)_{i j} \geq 0 \quad$ for all $i=1, \ldots, N_{0}$;

(2) $M_{i j} \geq 0 \quad$ for all $i=1, \ldots, N_{0}, j=1, \ldots, N$;

(3) $\sum_{j=1}^{N} M_{i j}=: m_{i}>0 \quad$ for all $i=1, \ldots, N_{0}$;

(4) $\mathbf{A}\left(\mathbf{u}^{n+1}\right)_{i j} \leq 0 \quad\left(i \neq j, \quad i=1, \ldots, N_{0}, j=1, \ldots, N\right)$;

(5) $\mathbf{B}\left(\mathbf{u}^{n}\right)_{i i} \geq 0 \quad\left(i=1, \ldots, N_{0}\right)$.

Proof. We follow the proof of Theorem 5.1. Statements (1)-(3) follow from it immediately, since (as seen obviously from its proof) the new growth conditions only affect the last two properties.

To prove properties (4)-(5), instead of $u_{h}$ in the arguments, we must consider the functions $u^{n+1}$ (for $\mathbf{A}$ ) and $u^{n}$ (for $\mathbf{B}$ ) that have the coefficient vectors $\mathbf{u}^{n+1}$ and $\mathbf{u}^{n}$, respectively. The derivations below then follow the proof of the scalar case [13] with a proper adaptation.

(4) Since we now have (7) instead of (69), the first estimate in (86) is replaced by

$$
\int_{\Omega} r_{k_{0} k_{0}}\left(x, t, u^{n+1}\right) \varphi_{p} \varphi_{q} \leq \int_{\Omega}\left(\alpha_{1}+\beta_{1}\left|u^{n+1}\right|^{p_{1}-2}\right) \varphi_{p} \varphi_{q} \leq \alpha_{1} \operatorname{meas}_{d}\left(\Omega_{p q}\right)+\beta_{1} \int_{\Omega_{p q}}\left|u^{n+1}\right|^{p_{1}-2} .
$$


Here the first term is bounded by $\alpha_{1} c_{3} h^{d}$ as before. To estimate the second term, we use Hölder's inequality:

$$
\int_{\Omega_{p q}}\left|u^{n+1}\right|^{p_{1}-2} \leq\left\|u^{n+1}\right\|_{L^{p_{1}\left(\Omega_{p q}\right)}}^{p_{1}-2}\|1\|_{L^{p_{1}\left(\Omega_{p q}\right)}}^{2}
$$

where $\left\|u^{n+1}\right\|_{L^{p_{1}\left(\Omega_{p q}\right)}}:=\left(\int_{\Omega_{p q}}\left|u^{n+1}\right|^{p_{1}}\right)^{\left(1 / p_{1}\right)}$ and $\left|u^{n+1}\right|$ stands for the Euclidean length of the values of vector function $u^{n+1}$. For the first factor, we use Lemma 5.2 (2) to find that

$$
\left\|u^{n+1}\right\|_{L^{p_{1}}\left(\Omega_{p q}\right)}^{p_{1}-2} \leq\left\|u^{n+1}\right\|_{L^{p_{1}}(\Omega)}^{p_{1}-2} \leq K_{p_{1}, \Omega}^{p_{1}-2}
$$

${ }_{462}$ The second factor satisfies, by (85), $\|1\|_{L^{p_{1}\left(\Omega_{p q}\right)}}^{2}=\left(\operatorname{meas}_{d}\left(\Omega_{p q}\right)\right)^{2 / p_{1}} \leq c_{3}^{\frac{2}{p_{1}}} h^{\frac{2 d}{p_{1}}} \equiv c_{3}^{\frac{2}{p_{1}}} h^{d-\gamma_{1}}$ with

$$
\gamma_{1}:=d-\frac{2 d}{p_{1}}<2
$$

since from Assumption 2.1 (A5) we have $\frac{2 d}{p_{1}}>d-2$. Hence $\int_{\Omega_{p q}}\left|u^{n+1}\right|^{p_{1}-2} \leq K_{p_{1}, \Omega}^{p_{1}-2} c_{3}^{\frac{2}{p_{1}}} h^{d-\gamma_{1}}$ and altogether,

$$
\int_{\Omega} r_{k_{0} k_{0}}\left(x, t, u^{n+1}\right) \varphi_{p} \varphi_{q} \leq \alpha_{1} c_{3} h^{d}+\beta_{1} K_{p_{1}, \Omega}^{p_{1}-2} c_{3}^{\frac{2}{p_{1}}} h^{d-\gamma_{1}}
$$

Similarly,

$$
\int_{\Gamma_{N}} z_{k_{0} k_{0}}\left(x, t, u^{n+1}\right) \varphi_{p} \varphi_{q} \leq \alpha_{2} c_{2} h^{d-1}+\beta_{2} \int_{\Gamma_{p q}}\left|u^{n+1}\right|^{p_{2}-2}
$$

and here we can use Assumption 5.3 (B4) and (72) to have

$$
\begin{gathered}
\int_{\Gamma_{p q}}\left|u^{n+1}\right|^{p_{2}-2} \leq\left\|u^{n+1}\right\|_{L^{p_{2}\left(\Gamma_{p q}\right)}}^{p_{2}-2}\|1\|_{L^{p_{2}\left(\Gamma_{p q}\right)}}^{2} \leq\left\|u^{n+1}\right\|_{L^{p_{2}\left(\Gamma_{N}\right)}}^{p_{2}-2}\left(\text { meas }_{d-1}\left(\Gamma_{p q}\right)\right)^{2 / p_{2}} \\
\leq M_{p_{2}}^{p_{2}-2} c_{2}^{\frac{2}{p_{2}}} h^{\frac{2(d-1)}{p_{2}}} \equiv M_{p_{2}}^{p_{2}-2} c_{2}^{\frac{2}{p_{2}}} h^{d-\gamma_{2}},
\end{gathered}
$$

${ }_{464}$ where $\Gamma_{p q}:=\partial \Omega_{p q} \cap \Gamma$ and

$$
\gamma_{2}:=d-\frac{2(d-1)}{p_{2}}<2
$$

since from Assumption 2.1 (A5) we have $\frac{2 d-2}{p_{2}}>d-2$. Summing up, using the above and (87), we obtain that $A\left(\mathbf{u}^{n+1}\right)_{i j}$ is bounded by

$c_{3} h^{d}\left[1+\theta \Delta t\left(-\frac{\mu_{0} K_{0}}{c_{3}} \frac{1}{h^{2}}+\alpha_{1}+\frac{c_{2} \alpha_{2}+c_{g r a d}\|\mathbf{w}\|_{L^{\infty}(\Omega)^{s}}}{c_{3} h^{\varrho \sigma}}+\frac{\beta_{1} K_{p_{1}, \Omega}^{p_{1}-2}}{c_{3}^{\frac{p_{1}-2}{p_{1}}} h^{\gamma_{1}}}+\frac{\beta_{2} c_{2}^{\frac{2}{p_{2}}} M_{p_{2}}^{p_{2}-2}}{c_{3} h^{\gamma_{2}}}\right)\right]$.

Since $h<h_{0}$ for $h_{0}$ defined in assumption (ii), it follows that we have a negative coefficient of $\theta \Delta t$ above, and from (95) we obtain that the expression in [...] is nonpositive, hence $A\left(\mathbf{u}^{h}\right)_{i j} \leq 0$. 
(5) For the considered implicit scheme, $\mathbf{B}\left(\mathbf{u}^{n}\right)$ coincides with the block mass matrix M, whose diagonal entries are positive.

From Theorem 5.3, one can derive the corresponding discrete maximum, minimum and nonnegativity preservation principles, similarly as in Lemma 5.1 and Theorem 5.2 in the sublinear case. Here we only formulate the discrete nonnegativity principle:

Corollary 5.3 Let the conditions of Theorem 5.3 hold, further, let $\hat{f}_{k} \geq 0, g_{k}^{h} \geq 0$ and $u_{k}^{(0), h} \geq 0$ for all $k=1, \ldots, s$. Then the fully discrete solution, obtained from (68), satisfies

$$
u_{i}^{n} \geq 0 \quad\left(n=0,1, \ldots, n_{T}, i=1, \ldots, N_{0}\right) .
$$

In addition, similarly to Remark 5.2, if we extend the solutions to $Q_{T}$ with values between those on the neighbouring time levels, e.g. with the method of lines, then we obtain that the coordinates of the discrete solution satisfy

$$
u_{k}^{h} \geq 0 \quad \text { on } Q_{T} \quad(k=1, \ldots, s) .
$$

Remark 5.3 In view of Corollary 5.3, it makes sense to pose problem (1)-(5) if its coefficients $q_{k}$ and/or $s_{k}$ are a priori defined only for nonnegative arguments for $u_{1}, \ldots, u_{s}$, since the described numerical solution only uses these values. This is the case for various real-life models with nonnegative unknown quantities, such as concentration etc. (If an actual inner numerical method still requires arbitrary values of $u_{1}, \ldots, u_{s}$, than one may define suitable extensions of $q_{k}$ and/or $s_{k}$.)

Remark 5.4 Similar comments are valid for the assumptions of Theorem 5.3 as in Remark 5.1. In particular, the lower bound in (95) for the space and time discretization steps is asymptotically of the form

$$
\Delta t \geq O\left(h^{2}\right)
$$

as $h \rightarrow 0$, and all the constants involved are easily computable. On the other hand, since we have considered the implicit scheme $\theta=1$ here, there is no corresponding upper bound as in Remark 5.1.

\subsection{Geometric properties of the space mesh}

In the above results, the condition on the space mesh to achieve the DMP has been property (93). We briefly summarize some geometric aspects of this condition.

The most direct way to satisfy (93) is to require the stricter property

$$
\nabla \varphi_{p} \cdot \nabla \varphi_{q} \leq-K_{0} h^{-2}
$$

pointwise on the common support of these basis functions. In view of well-known formulae (see e.g. $[2,5,27,41]$ ), the above condition has a nice geometric interpretation: in the case of simplicial meshes, it is sufficient if the employed mesh is uniformly acute [3, 27]. For practical constructions of such meshes see $[3,6,36]$ and references therein. In the case 
of bilinear elements, condition (98) is equivalent to the so-called strict non-narrowness of the meshes, see [19]. The case of prismatic finite elements in this context is treated in [16].

These conditions are sufficient but not necessary. For instance, for linear elements, some obtuse interior angles may occur in the simplices of the meshes, just as for linear problems (see e.g. [26]). Alternatively, one can require (98) only on a proper subpart of each intersection of supports [24]: let there exist subsets $\Omega_{p q}^{+} \subset \Omega_{p q}$ for all $p, q$ such that the basis functions satisfy

$$
\nabla \varphi_{p} \cdot \nabla \varphi_{q} \leq-\frac{c}{h^{2}}<0 \text { on } \Omega_{p q}^{+}, \quad \nabla \varphi_{q} \cdot \nabla \varphi_{p} \leq 0 \text { on } \Omega_{p q} \backslash \Omega_{p q}^{+},
$$

in which case the $\Omega_{p q}^{+}$must have asymptotically nonvanishing measure: $\frac{\text { meas }_{d}\left(\Omega_{p q}^{+}\right)}{\text {meas }_{d}\left(\Omega_{p q}\right)} \geq c_{3}>0$ for some constant $c_{3}$ independent of $p, q$. Clearly, these conditions ensure (93). These weaker conditions may allow in general easier refinement procedures (e.g. allow also right dihedral angles).

\section{$6 \quad$ Examples}

We give some examples of problems where the above DMP theorems yield new results. Let us recall here that the main conditions of the applied theorems are the relation $\Delta t=O\left(h^{2}\right)$ for the space and time mesh and the "acuteness" property (93) for the space mesh.

In all these examples, similarly as before, $\Omega$ stands for a bounded domain in $\mathbf{R}^{d}$ and $T>0$ is a given number, $\Gamma_{\text {int }}$ is a piecewise $C^{1}$ surface lying in $\Omega$, we denote $Q_{T}:=\left(\Omega \backslash \Gamma_{\text {int }}\right)$, and $[\cdot]_{\Gamma_{\text {int }}}$ denotes the jump (i.e., the difference of the limits from the two sides of the interface $\left.\Gamma_{i n t}\right)$ of a function.

\subsection{A single equation}

As a first trivial example, we mention that even for a single equation our results generalize those in [13] in two respects: first, one may now have nonsymmetric terms and interface conditions as well, second, the obtained DMP is now in a form directly analogous to the corresponding CMP.

Let us consider the equation

$$
\frac{\partial u}{\partial t}-\operatorname{div}(a(x, t, u, \nabla u) \nabla u)+\mathbf{w}(x, t) \cdot \nabla u+q(x, t, u)=f(x, t) \text { in } Q_{T},
$$

with boundary, interface and initial conditions analogous to (2)-(5) (in fact, one must simply drop the subscript $k$ therein). We impose Assumptions 2.1, which now reduce to the following simpler requirements. The domain and smoothness conditions (A1)-(A2) remain similar, just as the ellipticity condition $0<\mu_{0} \leq a(x, t, \xi, \eta) \leq \mu_{1}$ for the principal space term in (A3) and the coercivity conditions $\operatorname{div} \mathbf{w} \leq 0$ on $\Omega, \quad \mathbf{w} \cdot \nu \geq$ 0 on $\Gamma_{N},[\mathbf{w}]_{\Gamma_{i n t}}=0$ and $[\mathbf{w} \cdot \nu]_{\Gamma_{i n t}} \geq 0$ in (A4). Conditions (A5)-(A7) become 
much simpler: cooperativity has no meaning in this case, and the growth and diagonal dominance conditions together become

$$
0 \leq \frac{\partial q}{\partial \xi}(x, t, \xi) \leq \alpha_{1}+\beta_{1}|\xi|^{p_{1}-2}, \quad 0 \leq \frac{\partial s}{\partial \xi}(x, t, \xi) \leq \alpha_{2}+\beta_{2}|\xi|^{p_{2}-2} .
$$

Altogether, we just obtain a generalization of the problem in [13].

Then Lemma 5.1 holds together with its consequences. It is worth formulating what Theorem 5.2 yields for this case, as an analogue to (33):

Corollary 6.1 Let problem (99) and its FE discretization satisfy the conditions of Theorem 5.1. If the functions $u^{(0)}, g$ and $f$ are also continuous on the closure of their domains, then the discrete solution, obtained from (68), satisfies the following inequalities, where the notations of Lemma 5.1 are used:

(1) If $\hat{\gamma} \leq 0$, then $u_{i}^{n+1} \leq \max \left\{0, \max _{\bar{\Gamma}_{(n+1) \Delta t}^{D}} g^{h}, \max _{\bar{\Omega}} u^{(0), h}\right\}+(n+1) \Delta t \max \left\{0, \max _{\bar{Q}_{(n+1) \Delta t}} \hat{f}\right\}$.

(2) If $\hat{\gamma} \geq 0$, then $u_{i}^{n+1} \geq \min \left\{0, \min _{\bar{\Gamma}_{(n+1) \Delta t}^{D}} g^{h}, \min _{\bar{\Omega}} u^{(0), h}\right\}+(n+1) \Delta t \min \left\{0, \min _{\bar{Q}_{(n+1) \Delta t}} \hat{f}\right\}$.

(3) If $\hat{\gamma} \equiv 0$ or $\Gamma_{N} \cup \Gamma_{\text {int }}=\emptyset$, then both of the above inequalities are valid.

\subsection{Reaction-diffusion systems in chemistry}

\subsubsection{Reactions in a domain}

Certain reaction-diffusion processes in chemistry in a domain $\Omega \subset \mathbf{R}^{d}, d=2$ or 3 , are described by systems of the following form:

$$
\frac{\partial u_{k}}{\partial t}-b_{k} \Delta u_{k}+P_{k}\left(x, u_{1}, \ldots, u_{s}\right)=f_{k}(x, t) \text { in } Q_{T},
$$

with boundary and initial conditions

$$
u_{k}(x, t)=g_{k}(x, t) \quad \text { for } \quad(x, t) \in \Gamma_{D} \times[0, T],
$$

$$
b_{k} \frac{\partial u_{k}}{\partial \nu}=0 \quad \text { for } \quad(x, t) \in \Gamma_{N} \times[0, T], \quad u_{k}(x, 0)=u_{k}^{(0)}(x) \quad \text { for } \quad x \in \Omega \text {, }
$$

for all $k=1, \ldots, s$. The DMP for steady-states of such systems has been discussed in [24], now we consider the time-dependent case.

Here, for all $k$, the quantity $u_{k}$ describes the concentration of the $k$ th species, and $P_{k}$ is a polynomial which characterizes the rate of the reactions involving the $k$-th species. A common way to describe such reactions is the so-called mass action type kinetics $[17,18]$, which implies that $P_{k}$ has no constant term for any $k$, in other words, $P_{k}(x, 0) \equiv 0$ on $\Omega$ for all $k$. The function $f_{k} \geq 0$ describes a source independent of concentrations.

We consider system (101)-(103) under the following conditions, such that it becomes a special case of system (1)-(5). As pointed out later, such chemical models describe processes with cross-catalysis and strong autoinhibiton.

\section{Assumptions 6.2.1.}


(i) $\Omega$ is a bounded polytopic domain in $\mathbf{R}^{d}$, where $d=2$ or 3 , and $\Gamma_{N}, \Gamma_{D} \subset \partial \Omega$ are are disjoint open measurable subsets of $\partial \Omega$ such that $\partial \Omega=\bar{\Gamma}_{D} \cup \bar{\Gamma}_{N}$.

(ii) (Smoothness and growth.) For all $k, l=1, \ldots, s$, the functions $P_{k}$ are polynomials of arbitrary degree if $d=2$ or of degree at most 4 if $d=3$, and we have $P_{k}(x, 0) \equiv 0$ on $\Omega$. Further, $f_{k} \in L^{\infty}\left(Q_{T}\right), g_{k} \in L^{\infty}\left(\Gamma_{D} \times[0, T]\right)$ and $u_{k}^{(0)} \in L^{\infty}(\Omega)$.

(iii) (Ellipticity for the principal space term.) $b_{k}>0(k=1, \ldots, s)$ are given numbers.

(iv) (Cooperativity.) We have

$$
\frac{\partial P_{k}}{\partial \xi_{l}}(x, \xi) \leq 0 \quad\left(k, l=1, \ldots, s, k \neq l ; x \in \Omega, \xi \in \mathbf{R}^{s}\right) .
$$

(v) (Weak diagonal dominance w.r.t. rows and columns.) We have

$$
\sum_{l=1}^{s} \frac{\partial P_{k}}{\partial \xi_{l}}(x, \xi) \geq 0, \quad \sum_{l=1}^{s} \frac{\partial P_{l}}{\partial \xi_{k}}(x, \xi) \geq 0 \quad\left(k=1, \ldots, s ; x \in \Omega, \xi \in \mathbf{R}^{s}\right) .
$$

Similarly as in Remark 2.1, assumptions (104)-(105) now imply

$$
\frac{\partial P_{k}}{\partial \xi_{k}}(x, \xi) \geq 0 \quad\left(k=1, \ldots, s ; x \in \Omega, \xi \in \mathbf{R}^{s}\right) .
$$

Returning to the model described by system (101)-(103), the chemical meaning of the cooperativity (104) is cross-catalysis, whereas (106) means autoinhibiton. Cross-catalysis arises e.g. in gradient systems [35]. Condition (105) means that autoinhibition is strong enough to ensure both weak diagonal dominances.

By definition, the concentrations $u_{k}$ are nonnegative, therefore a proper numerical model must produce such numerical solutions. We can use Corollary 5.3 to obtain the required property:

Corollary 6.2 Let system (101)-(103) satisfy Assumptions 6.2.1, and assume that $u_{k}(., t) \in$ $W^{1, q}(\Omega)$ for some $q>2$ as in Assumptions 5.3 (B3). Let the FE discretization of the system satisfy the conditions of Theorem 5.3.

If $f_{k} \geq 0, g_{k}^{h} \geq 0$ and $u_{k}^{(0), h} \geq 0$ for all $k=1, \ldots, s$, then the discrete solution, obtained from (68), satisfies

$$
u_{i}^{n} \geq 0 \quad\left(n=0,1, \ldots, n_{T}, i=1, \ldots, N_{0}\right) .
$$

In addition, as mentioned after Corollary 5.3, if we extend the solutions to $Q_{T}$ with values between those on the neighbouring time levels, e.g. with the method of lines, then we obtain that the coordinates of the discrete solution satisfy

$$
u_{k}^{h} \geq 0 \quad \text { on } Q_{T} \quad(k=1, \ldots, s) .
$$

Remark 6.1 For such systems with only Dirichlet boundary conditions, more specific results on the preservation of invariant rectangles under FEM have been obtained in [8]. 


\subsubsection{Reactions localized on an interface}

A different type of reaction-diffusion process arises in some cases when the chemical reactions are localized on an interface, i.e. on a subsurface of the domain in 3D or on a curve in $2 \mathrm{D}$, see $[20,21]$ and the references therein. If one consideres such time-dependent systems, then the problem can be described as follows, where $\Omega \subset \mathbf{R}^{d}$ is a domain in $d=2$ or 3 :

$$
\frac{\partial u_{k}}{\partial t}-b_{k} \Delta u_{k}=f_{k}(x, t) \text { in } Q_{T},
$$

with boundary, interface and initial conditions

$$
u_{k}(x, t)=g_{k}(x, t) \quad \text { for } \quad(x, t) \in \partial \Omega \times[0, T],
$$

$$
\left[u_{k}\right]_{\Gamma_{i n t}}=0 \text { and }\left[b_{k} \frac{\partial u_{k}}{\partial \nu}+S_{k}\left(x, u_{1}, \ldots, u_{s}\right)\right]_{\Gamma_{i n t}}=0 \quad \text { for }(x, t) \in \Gamma_{i n t} \times[0, T] \text {, }
$$

$$
u_{k}(x, 0)=u_{k}^{(0)}(x) \quad \text { for } \quad x \in \Omega
$$

for all $k=1, \ldots, s$.

Analogously to Assumptions 6.2.1, we now impose

\section{Assumptions 6.2.2.}

(i) $\Omega$ is a bounded polytopic domain in $\mathbf{R}^{d}$, where $d=2$ or 3 , and $\Gamma_{\text {int }}$ is a piecewise $C^{1}$ surface lying in $\Omega$.

(ii) (Smoothness and growth.) For all $k, l=1, \ldots, s$, the functions $S_{k}$ are polynomials of arbitrary degree if $d=2$ or of degree at most 2 if $d=3$, and we have $S_{k}(x, 0) \equiv 0$ on $\Omega$. Further, $f_{k} \in L^{\infty}\left(Q_{T}\right), g_{k} \in L^{\infty}(\partial \Omega \times[0, T])$ and $u_{k}^{(0)} \in L^{\infty}(\Omega)$.

(iii) (Ellipticity for the principal space term.) $b_{k}>0(k=1, \ldots, s)$ are given numbers.

(iv) (Cooperativity.) We have $\frac{\partial S_{k}}{\partial \xi_{l}}(x, \xi) \leq 0 \quad\left(k, l=1, \ldots, s, k \neq l ; x \in \Gamma_{i n t}, \xi \in \mathbf{R}^{s}\right)$.

(v) (Weak diagonal dominance w.r.t. rows and columns.) We have

$$
\sum_{l=1}^{s} \frac{\partial S_{k}}{\partial \xi_{l}}(x, \xi) \geq 0, \quad \sum_{l=1}^{s} \frac{\partial S_{l}}{\partial \xi_{k}}(x, \xi) \geq 0 \quad\left(k=1, \ldots, s ; x \in \Gamma_{\text {int }}, \xi \in \mathbf{R}^{s}\right) .
$$

Similarly to the previous subsection, assumptions (iv)-(v) imply the analogue of (106), and the chemical meaning for the localized reactions is cross-catalysis and autoinhibition, the latter being strong enough to ensure both weak diagonal dominances.

We can repeat Corollary 6.2, by replacing Assumptions 6.2.1 by Assumptions 6.2.2, to obtain that $u_{i}^{n} \geq 0 \quad\left(n=0,1, \ldots, n_{T}, i=1, \ldots, N_{0}\right)$, and, by a proper extension of $u^{h}$ to $Q_{T}$, that $u_{k}^{h} \geq 0$ on $Q_{T} \quad(k=1, \ldots, s)$. 


\subsection{Transport problems}

Systems describing transport processes generally involve reaction, diffusion and convection (advection) terms. (Some other possible terms can be mathematically included in the last, zeroth-order reaction terms.) Let us first consider the case of reactions in the whole domain, see, e.g., [42].

The mathematical model of such processes is a modification of (101) if a first order term is added to describe convection. Let us therefore consider the system of equations

$$
\frac{\partial u_{k}}{\partial t}-b_{k} \Delta u_{k}+\mathbf{w}_{k}(x, t) \cdot \nabla u_{k}+P_{k}\left(x, u_{1}, \ldots, u_{s}\right)=f_{k}(x, t) \text { in } Q_{T}
$$

$(k=1, \ldots, s)$ with the boundary and initial conditions (102)-(103). We study this system under conditions such that it becomes a special case of system (1)-(5). For this, we only need to add the corresponding part of Assumption 2.1 (A4) to the previously studied properties:

Assumptions 6.3.1. Let Assumptions 6.2.1 hold, and let $\operatorname{div} \mathbf{w}_{k} \leq 0$ on $\Omega$ and $\mathbf{w}_{k} \cdot \nu \geq 0$ on $\Gamma_{N} \quad(k=1, \ldots, s)$.

As pointed out above, Assumptions 6.2.1 mean that the described chemical process is cross-catalyc with suitably strong autoinhibiton. Further, in many cases the convective terms are divergence-free (e.g. if they arise from a related Stokes system): $\operatorname{div} \mathbf{w}_{k}=0$, i.e. the first property of $\mathbf{w}_{k}$ holds. The inequality $\mathbf{w}_{k} \cdot \nu \geq 0$ on $\Gamma_{N}$ means that Neumann conditions are prescribed on the outflow boundary.

Similarly as before, the concentrations $u_{k}$ are nonnegative, therefore the numerical model must produce such numerical solutions. We can repeat Corollary 6.2 , by replacing Assumptions 6.2.1 by Assumptions 6.3.1, to obtain that $u_{i}^{n} \geq 0 \quad\left(n=0,1, \ldots, n_{T}, i=\right.$ $\left.1, \ldots, N_{0}\right)$, and, by a proper extension of $u^{h}$ to $Q_{T}$, that $u_{k}^{h} \geq 0$ on $Q_{T} \quad(k=1, \ldots, s)$.

Second, for transport processes we can also consider the case when the chemical reactions are localized on an interface. Then we only have uncoupled nonsymmetric equations such that the reactions $P_{k}\left(x, u_{1}, \ldots, u_{s}\right)$ are missing from (111), and they instead appear in the interface conditions as in subsection 6.2.2, i.e. the side conditions are (108)-(110). In this case Assumptions 6.2 .2 are completed with the conditions $\left[\mathbf{w}_{k}\right]_{\Gamma_{\text {int }}}=0$ and $\left[\mathbf{w}_{k} \cdot \nu\right]_{\Gamma_{\text {int }}} \geq 0 \quad(k=1, \ldots, s)$, and provide the desired nonnegativity if these assumptions replace Assumptions 6.2.1 in Corollary 6.2.

\subsection{Population systems and reactions proportional to species}

Certain systems in population dynamics can be written in the form

$$
\left\{\begin{array}{l}
\frac{\partial u_{1}}{\partial t}-b_{1} \Delta u_{1}=u_{1} M_{1}\left(u_{1}, u_{2}\right) \\
\frac{\partial u_{2}}{\partial t}-b_{2} \Delta u_{2}=u_{2} M_{2}\left(u_{1}, u_{2}\right)
\end{array}\right.
$$


where $u_{1}, u_{2}$ denote the amounts of two species distributed continuously in a plane region $\Omega$, see e.g. [8]. The simple boundary and initial conditions

$$
u_{k}=g_{k} \quad \text { on } \partial \Omega \times[0, T], \quad u_{k}(., 0)=u_{k}^{(0)} \quad \text { on } \Omega \quad(k=1,2)
$$

are imposed. Such a system can also describe a chemical reaction as in subsection 6.2 if the reaction rates are proportional to the quantity of the species. Here we will use the population terminology. If the species live in symbiosis, then

$$
\partial_{2} M_{1} \geq 0 \quad \text { and } \quad \partial_{1} M_{2} \geq 0 \text {. }
$$

System (112) falls into the type (1) where

$$
q_{1}\left(\xi_{1}, \xi_{2}\right)=-\xi_{1} M_{1}\left(\xi_{1}, \xi_{2}\right) \quad \text { and } \quad q_{2}\left(\xi_{1}, \xi_{2}\right)=-\xi_{2} M_{2}\left(\xi_{1}, \xi_{2}\right)
$$

and $f_{1} \equiv f_{2} \equiv 0$. Most of Assumptions 2.1 are trivially satisfied in a natural way. Namely, let us impose

Assumptions 6.4.1. $\Omega$ is a bounded polygonal domain in $\mathbf{R}^{2}$ and $b_{1}, b_{2}>0$ are given numbers. Further, $g_{1}, g_{2} \in C(\partial \Omega \times[0, T]), u_{1}^{(0)}, u_{2}^{(0)} \in C(\bar{\Omega}), \quad M_{1}, M_{2} \in C^{1}\left(\mathbf{R}^{2}\right)$ and they can grow at most with polynomial rate with $\xi_{1}, \xi_{2}$.

These assumptions imply that (A1)-(A5) of Assumptions 2.1 are satisfied. The cooperativity (A6) follows from (114), since by Remark 5.3 we may only consider nonnegative values of $\xi_{k}$. In view of Theorem 5.3 that we want to use, it suffices to fulfil the weak diagonal dominances (90). Before giving a condition, we recall the property in Remark 2.1, necessary for diagonal dominance. This expresses that the $q_{k}$ grow along with their quantity, and for (115), it amounts to $\partial_{i}\left(\xi_{i} M_{i}\left(\xi_{1}, \xi_{2}\right)\right) \leq 0(i=1,2)$ for all $\xi_{1}, \xi_{2}$, where $\partial_{i}:=\frac{\partial}{\partial \xi_{i}}$. The exact condition for diagonal dominance is a strengthened version of this:

Proposition 6.1 The functions (115) satisfy (90) if and only if for all $i, j, k=1,2$ and $\xi_{1}, \xi_{2}>0$

$$
\partial_{i}\left(\xi_{i} M_{i}\left(\xi_{1}, \xi_{2}\right)\right) \leq-\xi_{j} \partial_{k} M_{j}\left(\xi_{1}, \xi_{2}\right) \quad(j \neq k) .
$$

Proof. For brevity, we omit the variables $\left(\xi_{1}, \xi_{2}\right)$ after $M_{i}$. The result follows by checking four elementary relations for (115):

$$
\begin{aligned}
\partial_{1} q_{1}+\partial_{2} q_{1} \geq 0 & \Leftrightarrow \partial_{1}\left(\xi_{1} M_{1}\right) \leq-\xi_{1} \partial_{2} M_{1}, \\
\partial_{1} q_{2}+\partial_{2} q_{2} \geq 0 & \Leftrightarrow \partial_{2}\left(\xi_{2} M_{2}\right) \leq-\xi_{2} \partial_{1} M_{2}, \\
\partial_{1} q_{1}+\partial_{1} q_{2} \geq 0 & \Leftrightarrow \partial_{1}\left(\xi_{1} M_{1}\right) \leq-\xi_{2} \partial_{1} M_{2}, \\
\partial_{2} q_{1}+\partial_{2} q_{2} \geq 0 & \Leftrightarrow \partial_{2}\left(\xi_{2} M_{2}\right) \leq-\xi_{1} \partial_{2} M_{1} .
\end{aligned}
$$


Remark 6.2 For instance, the functions (115) sometimes have the form

$$
q_{i}\left(\xi_{1}, \xi_{2}\right)=G_{i} \xi_{i}-\xi_{i} \xi_{j} h_{i}\left(\xi_{1}, \xi_{2}\right), \quad \text { then } \quad M_{i}\left(\xi_{1}, \xi_{2}\right)=-G_{i}+\xi_{j} h_{i}\left(\xi_{1}, \xi_{2}\right)
$$

( $i=1,2, i \neq j)$, where $G_{i}>0$ is the birth-death rate and $h_{i}$ is a factor for the coexistence of the species. For instance, some Lotka-Volterra type systems can fall into this type. Assume that the rates $h_{i}$ are small for large populations, in particular, that $\left|\partial_{k} h_{i}\left(\xi_{1}, \xi_{2}\right)\right| \leq \frac{c_{1}}{1+\xi_{1}^{2}+\xi_{2}^{2}}$. In this case an elementary calculation shows that if $c_{1}$ is so small that $c_{1}(1+2 \sqrt{2}) \leq \min \left(G_{1}, G_{2}\right)$, then $M_{i}$ satisfy (116).

Now we can use Corollary 5.3 to obtain the required nonnegativity for the numerically computed populations:

Corollary 6.3 Let system (112)-(113) satisfy (114), Assumptions 6.4.1 and (116). Assume further that $u_{k}(., t) \in W^{1, q}(\Omega)(k=1,2)$ for some $q>2$ as in Assumptions 5.3 (B3). Let the FE discretization of the system satisfy the conditions of Theorem 5.3.

If $g_{1}^{h}, g_{2}^{h} \geq 0$ and $u_{1}^{(0), h}, u_{2}^{(0), h} \geq 0$, then the discrete solution, obtained from (68), satisfies

$$
u_{i}^{n} \geq 0 \quad\left(n=0,1, \ldots, n_{T}, i=1, \ldots, N_{0}\right) .
$$

Further, by a proper extension of $u^{h}$ to $Q_{T}$, we have $u_{1}^{h}, u_{2}^{h} \geq 0$ on $Q_{T}$.

\section{References}

[1] A. Berman, R. Plemmons, Nonnegative Matrices in Mathematical Sciences, Academic Press, New York, 1979.

[2] J. Brandts, S. Korotov, M. KřÍžEk, Dissection of the Path-Simplex in $\mathbf{R}^{n}$ into $n$ Path-Subsimplices, Linear Algebra Appl. 421 (2007), pp. 382-393.

[3] J. Brandts, S. Korotov, M. Křížek, J. Šolc, On Nonobtuse Simplicial Partitions, SIAM Rev. 51 (2009), No. 2, pp. 317-335.

[4] P. G. Ciarlet, Discrete Maximum Principle for Finite Difference Operators, Aequationes Math. 4 (1970), pp. 338-352.

[5] P. G. Ciarlet, P. A. Raviart, Maximum Principle and Uniform Convergence for the Finite Element Method, Comput. Methods Appl. Mech. Engrg. 2 (1973), pp. $17-31$.

[6] D. Eppstein, J. M. Sullivan, A. ÜngÖR, Tiling space and slabs with acute tetrahedra, Comput. Geom. 27 (2004), no. 3, 237-255.

[7] L.C. Evans, A strong maximum principle for parabolic systems in a convex set with arbitrary boundary, Proc. Amer. Math. Soc. 138 (2010), No. 9, pp. 3179-3185. 
[8] D. J. Estep, M. G. Larson, R. D. Williams, Estimating the error of numerical solutions of systems of reaction-diffusion equations, Mem. Amer. Math. Soc. 146 (2000), no. 696, viii+109 pp.

[9] I. Faragó, R. Horváth, Discrete Maximum Principle and Adequate Discretizations of Linear Parabolic Problems, SIAM J. Sci. Comput. 28 (2006), pp. 2313-2336.

[10] I. Faragó, R. Horváth, Continuous and Discrete Parabolic Operators and their Qualitative Properties, IMA J. Numer. Anal. 29 (2009), pp. 606-631.

[11] I. Faragó, R. Horváth, S. Korotov, Discrete Maximum Principles for FE Solutions of Nonstationary Diffusion-Reaction Problems with Mixed Boundary Conditions, Numer. Methods Part. Diff. Eqns. 27 (2011), 702-720.

[12] I. Faragó, J. Karátson, Numerical Solution of Nonlinear Elliptic Problems via Preconditioning Operators. Theory and Applications. Advances in Computation, Volume 11, NOVA Science Publishers, New York, 2002.

[13] I. Faragó, J. Karátson, S. Korotov, Discrete maximum principles for the FEM solution of some nonlinear parabolic problems, Electr. Trans. Numer. Anal. 36 (2009-2010), pp. 149-167.

[14] A. Friedmann, Partial Differential Equations of Parabolic Type, Prentice-Hall, Englewood Cliffs, 1964.

[15] H. FujII, Some Remarks on Finite Element Analysis of Time-Dependent Field Problems, Theory and Practice in Finite Element Structural Analysis, Univ. Tokyo Press, Tokyo (1973), pp. 91-106.

[16] A. Hannukainen, S. Korotov, T. Vejchodský, Discrete Maximum Principles for FE Solutions of the Diffusion-Reaction Problem on Prismatic Meshes, J. Comput. Appl. Math. 226 (2009), pp. 275-287.

[17] V. Hárs, J. Tóth, On the inverse problem of reaction kinetics, In: Qualitative Theory of Differential Equations (Szeged, Hungary, 1979), Coll. Math. Soc. János Bolyai 30, ed. M. Farkas, North-Holland - János Bolyai Mathematical Society, Budapest, 1981, pp. 363-379.

[18] F. Horn, R. Jackson, General mass action kinetics, Arch. Rat. Mech. Anal. 47 (1972), 81-116.

[19] R. Horváth, Sufficient Conditions of the Discrete Maximum-Minimum Principle for Parabolic Problems on Rectangular Meshes, Comput. Math. Appl. 55 (2008), pp. 2306-2317.

[20] J. D. Kandilarov, A monotone iterative method for numerical solution of diffusion equations with nonlinear localized chemical reactions, in: Numerical Methods and Applications, Lecture Notes in Comput. Sci. 4310, 615-622 (2007); Springer. 
[21] J. D. Kandilarov, L. G. Vulkov, Analysis of immersed interface difference schemes for reaction-diffusion problems with singular own sources, Comput. Methods Appl. Math. 3 (2003), no. 2, 253-273 (electronic).

[22] J. Karátson, S. Korotov, Discrete Maximum Principles for Finite Element Solutions of Nonlinear Elliptic Problems with Mixed Boundary Conditions, Numer. Math. 99 (2005), pp. 669-698.

[23] J. Karátson, S. Korotov, Discrete maximum principles for FEM solutions of some nonlinear elliptic interface problems, Int. J. Numer. Anal. Modelling 6, 2009, pp. 1-16.

[24] J. Karátson, S. Korotov, An algebraic discrete maximum principle in Hilbert space with applications to nonlinear cooperative elliptic systems, SIAM Numer. Anal. 47 (2009), No. 4, pp. 2518-2549.

[25] H. B. Keller, The Numerical Solution of Parabolic Partial Differential Equations, In: Mathematical Methods for Digital Computers (eds. A. Ralston, H.S. Wilf), New York, 1960, pp. 135-143.

[26] S. Korotov, M. KŘížek, P. Neittaanmäki, Weakened Acute Type Condition for Tetrahedral Triangulations and the Discrete Maximum Principle, Math. Comp. 70 (2001), pp. 107-119.

[27] M. KŘížEK, Qun Lin, On Diagonal Dominance of Stiffness Matrices in 3D, EastWest J. Numer. Math. 3 (1995), pp. 59-69.

[28] O. A. Ladyzenskaja, V. A. Solonnikov, N. N. Ural'ceva, Linear and Quasilinear Equations of Parabolic Type, Translations of Mathematical Monographs, Vol. 23, Amer. Math. Soc., Providence, R.I., 1968.

[29] C. V. PAO, Nonlinear parabolic and elliptic equations, Plenum Press, New York, 1992.

[30] C. V. PAO, Numerical analysis of coupled systems of nonlinear parabolic equations, SIAM J. Numer. Anal. 36 (1999), no. 2, 393-416

[31] T. Stys, T. Motsumi, O. Daman, On the maximum principle and its application to diffusion equations, Numer. Methods Part. Diff. Eqns. 23 (2007), pp. 60-72.

[32] V. Thomée, Galerkin Finite Element Methods for Parabolic Problems, Springer, Berlin, 1997.

[33] V. Thomée, Chen, C.M., The lumped mass finite element method for a parabolic problem, J. Aust. Math. Soc., Ser. B, 26 (1985), pp. 329-354.

[34] V. Thomée, Wahlbin L. B., On the existence of maximum principles in parabolic finite element equations, Math. Comp. 77 (2008), No. 261, pp. 11-19. 
[35] J. Tóтн, Gradient systems are cross-catalytic, Reaction Kinetics and Catalysis Letters 12 (3) (1979), 253-257.

[36] E. VanderZee, A. N. Hirani, V. Zharnitsky, D. Guoy, A dihedral acute triangulation of the cube, Comput. Geom. 43, No. 5, 445-452 (2010).

[37] R. VArga, Matrix Iterative Analysis, Prentice Hall, New Jersey, 1962.

[38] T. VejchodskÝ, P. Solín, Discrete maximum principle for higher-order finite elements in 1D, Math. Comp. 76 (2007), pp. 1833-1846.

[39] H. Weinberger, Invariant sets for weakly coupled parabolic and elliptic systems, Rend. Mat., VI. Ser. 8 (1975), pp. 295-310.

[40] X. WANG, A remark on strong maximum principle for parabolic and elliptic systems, Proc. Amer. Math. Soc. 109 (1990), No.2, pp. 343-348.

[41] J. Xu, L. ZIKatanov, A Monotone finite element scheme for convection-diffusion equations, Math. Comp. 68 (1999), pp. 1429-1446.

[42] Zlatev, Z., Computer treatment of large air pollution models, Kluwer Academic Publishers, Dordrecht-Boston-London, 1995. 